\title{
البحثث الأول:
}

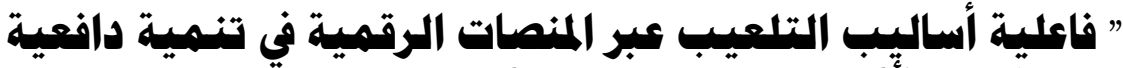

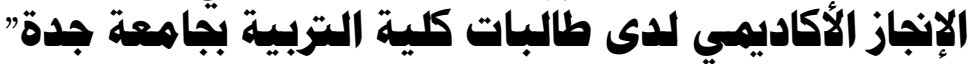

$$
\begin{aligned}
& \text { إلحكاك : }
\end{aligned}
$$

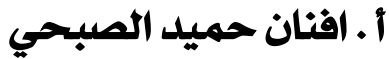

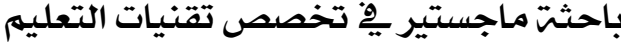

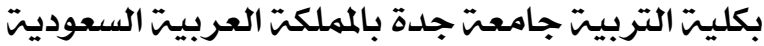

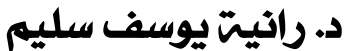

$$
\begin{aligned}
& \text { أستاذ تقنيات التعليم المثارك بكليت بليت التربيت } \\
& \text { جامعتَ جدة بالمملكت العربيت السعوديني }
\end{aligned}
$$





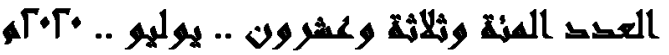

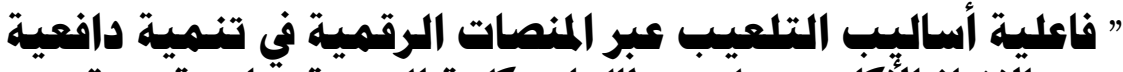

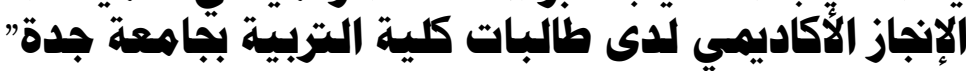

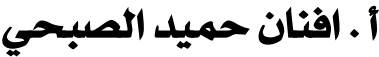

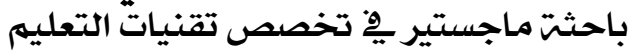 بكليت التربيت جامعتر جدة بالمملكت العربيت السعوديتة

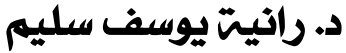

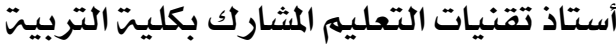 \\ جامعتَ جدة بالمملكت العربيت السعوديتي}

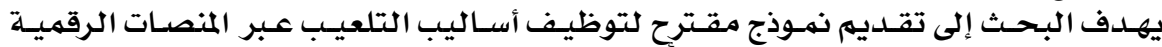

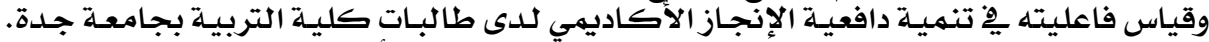

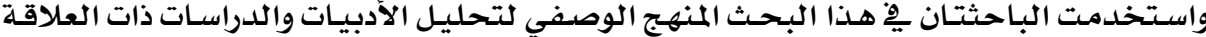

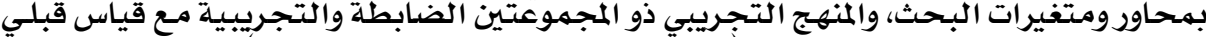

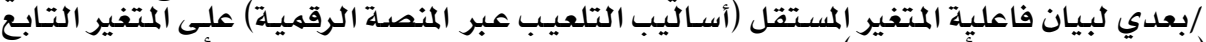

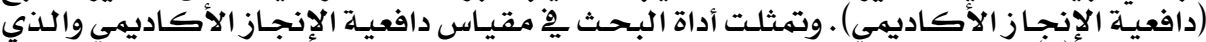

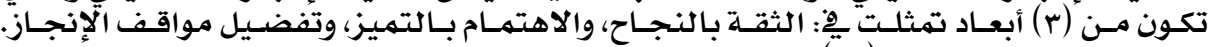

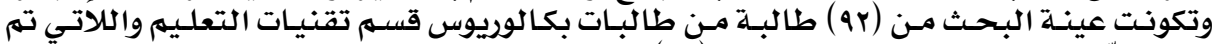

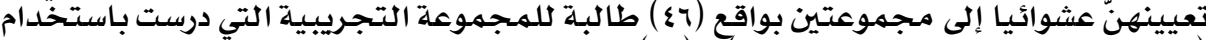

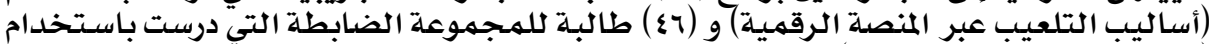

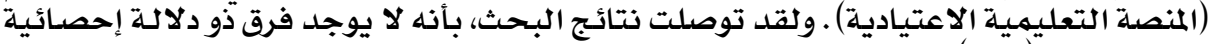

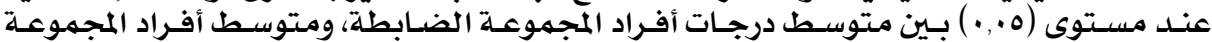

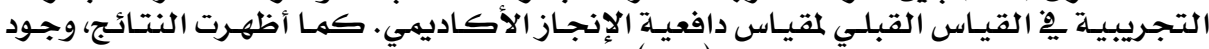

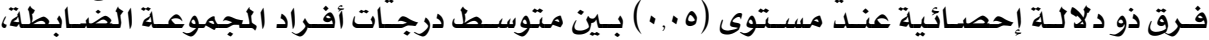

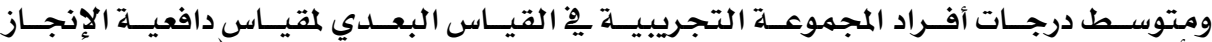

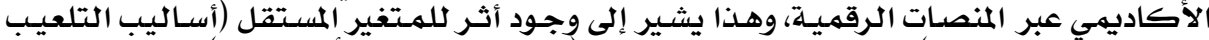

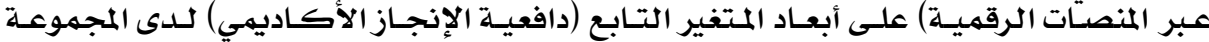

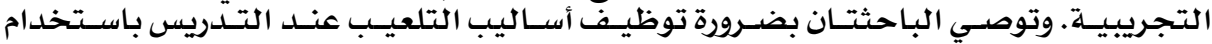

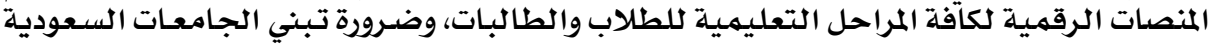

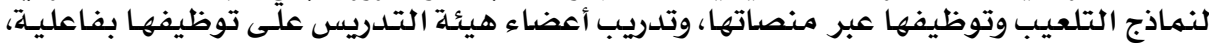

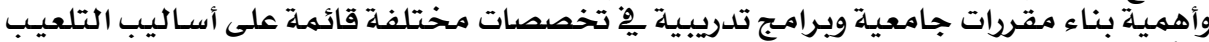

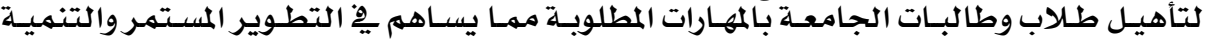

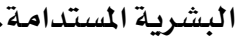

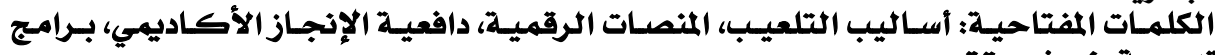
تدريبية، نموذج مقترح.

"Proposed Model to Employ Some Gamification Techniques Via Massive Open Online Courses (MOOCs) and its Eeffectiveness in the Developing of Academic Achievement Motivation toward Learning among Students in the Faculty of Education at Jeddah University"

Afnan Hameed Alsubhi \& Dr.Rania Youssef Selim $\underline{\text { Abstract }}$

This research aims to propose a model to employ some gamification techniques via massive open online courses and to evaluate its effectiveness in 


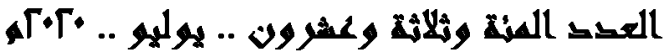

developing academic achievement motivation toward learning among students in the faculty of Education at University of Jeddah. The researcher used the descriptive approach to analyse literature relevant to research themes and variables, and she used the experimental and control groups, with pre-and post-measures to demonstrate the effect of the independent variable (i.e. gamification techniques via MOOC) on the dependent variable (academic achievement motivation). The research tool was academic achievement motivation measurement that consist of 3 aspects which are: confidence in success, interest in excellence, and preference for achievement experiences. The research was carried out on a sample of (92) students who are studying for bachelor degree of educational technology, and they were randomly divided into two groups; (46) of them were assigned to the experimental group who was studying using gamification techniques via MOOC. The other (46) were allocated into the control group, who was studying using the traditional MOOC. The research results showed that there is no statistically significant difference at (0.05) level between average mean scores of the control group and the experimental group in the pre-measurement of academic achievement motivation. The research results also revealed that there is a statistically significant difference at (0.05) level between average mean scores of the control group and the average scores of the experimental group in the postmeasurement of academic achievement motivation, which indicates the effect of the independent variable on the aspects of dependant variable. The researcher recommends that gamification techniques should be employed in teaching, by using MOOCs for all educational stages. The researcher also recommends Saudi universities to adopt gamification models and employ them through their platforms, train their faculty members to effectively employ it. The researcher also emphasizes the importance of developing curricula and training programs in several fields based on gamification techniques to qualify university students with needed skills to extend the sustainable development. Keywords: gamification techniques, MOOCs, academic achievement motivation, training programs, proposed model.

مِّ ظل الانفٍ

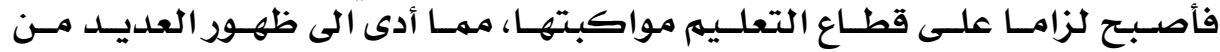

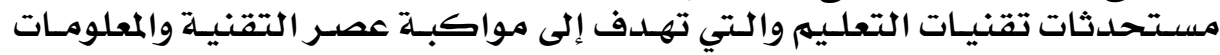

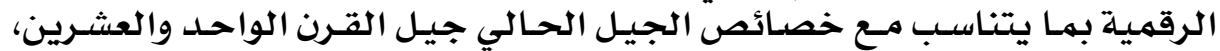

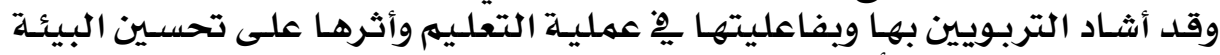

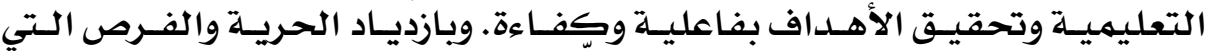

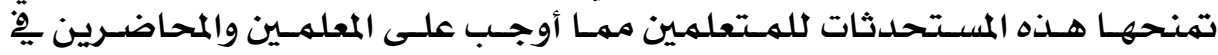

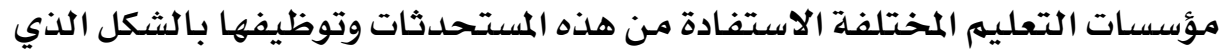

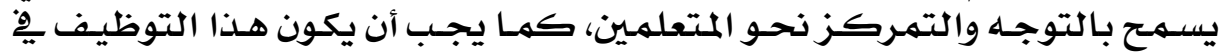

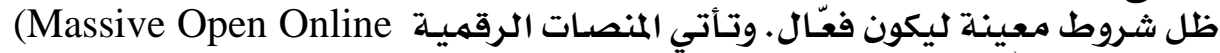
أو (MOUCses)

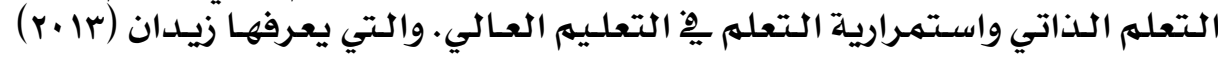




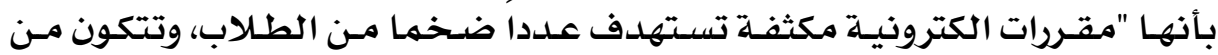

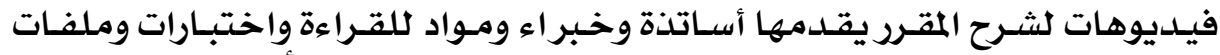

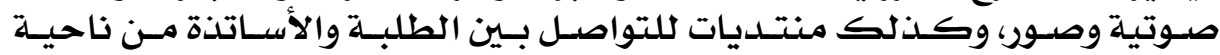

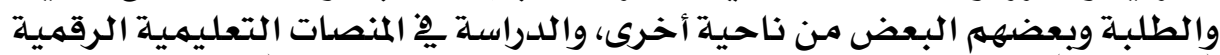
(MOOCs)

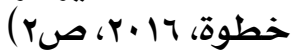

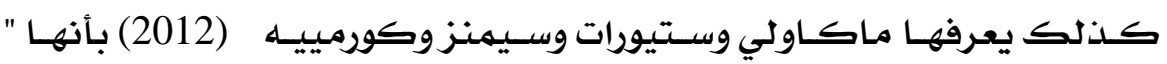

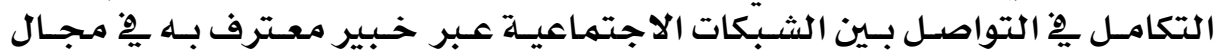

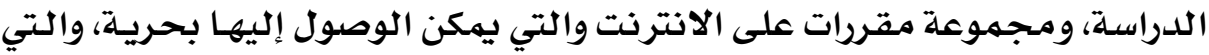

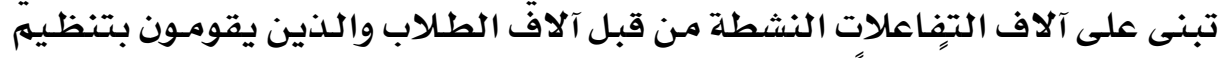

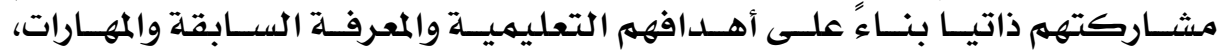

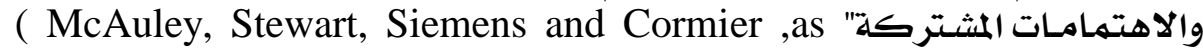
cited in Rodriguez ,2012,p.3).

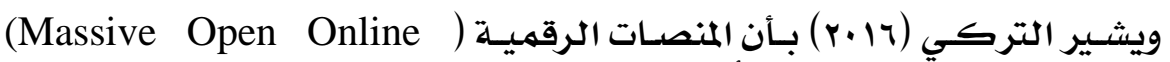

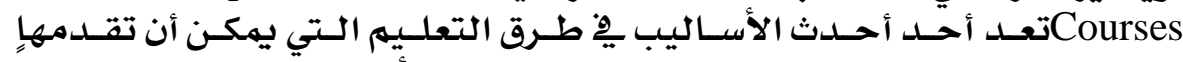

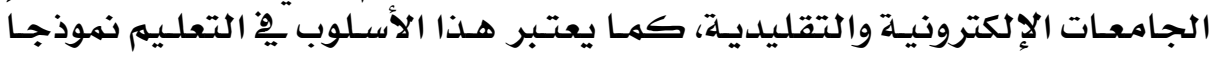

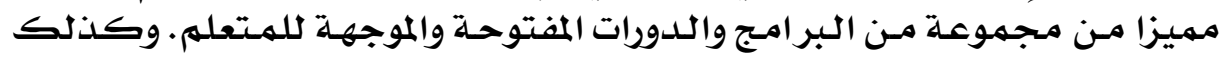

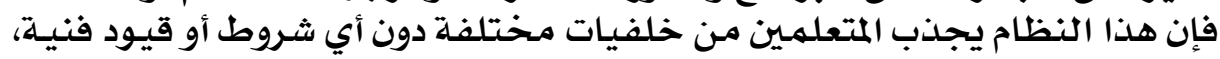

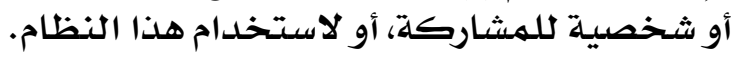

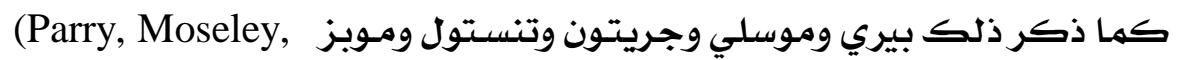
Gretton, Tunstall \& Mobbs,2016)

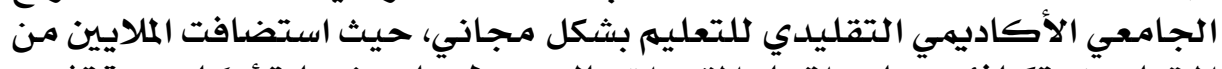

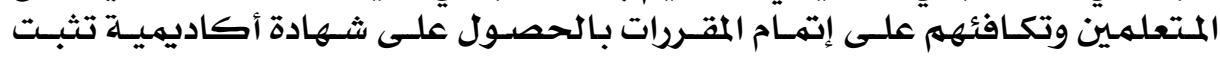

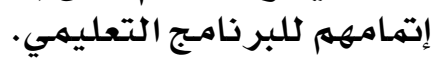

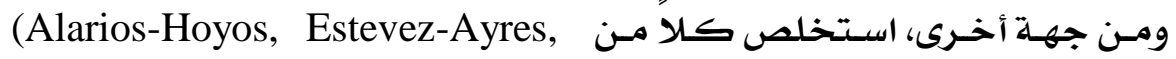

Terras and ؛Sanagustin, Frenandez-Panadero, Kloos and Perez,2017 Ramsay,2015;

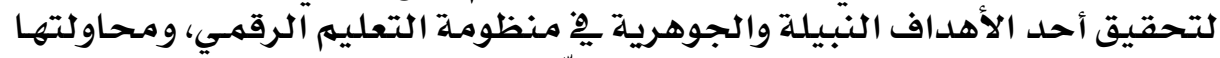

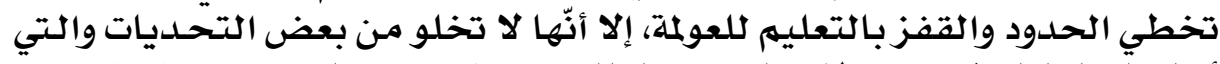

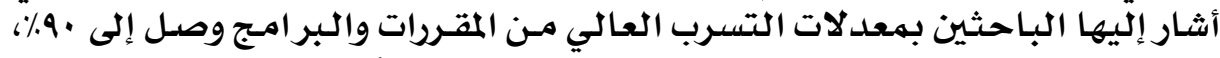

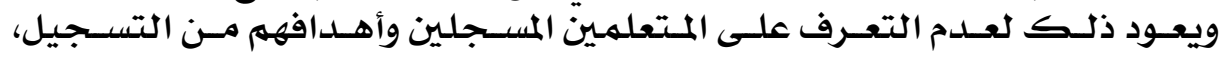

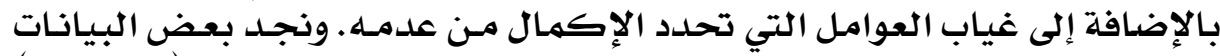

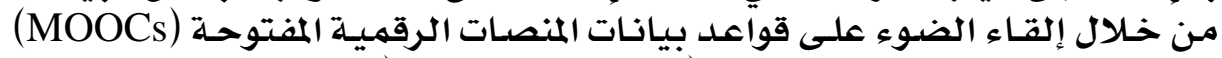

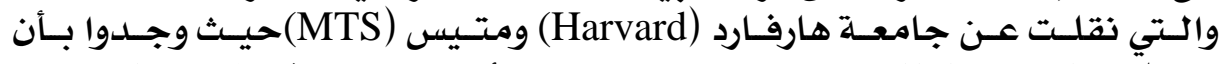

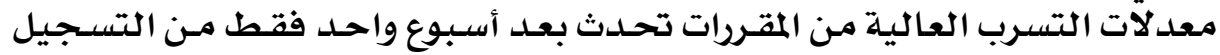




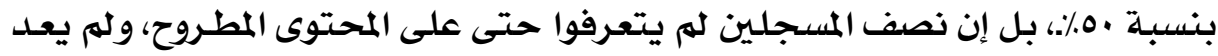

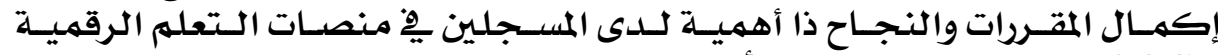

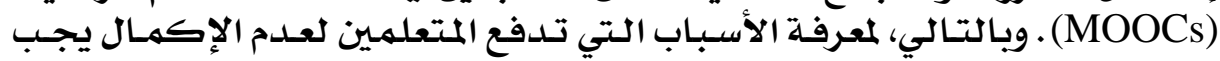

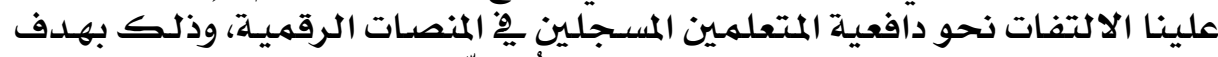

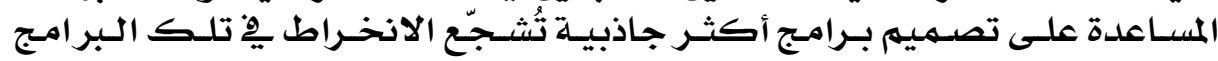

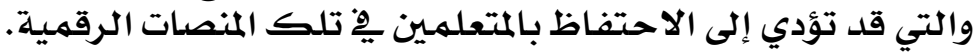

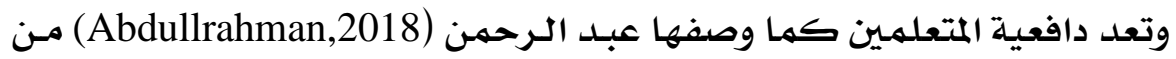

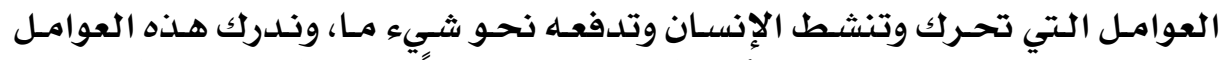

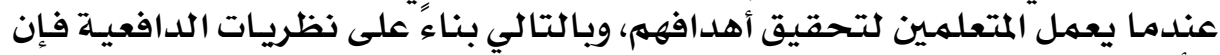

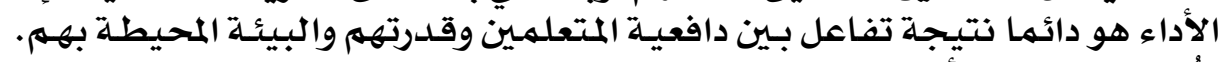

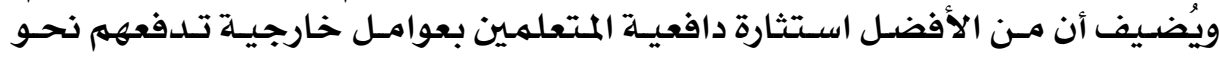

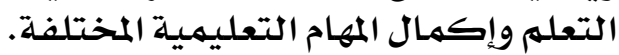

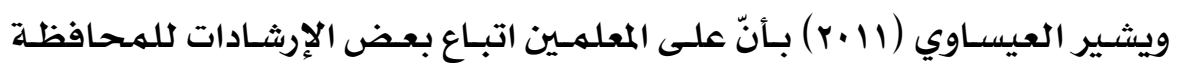

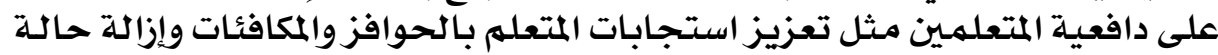

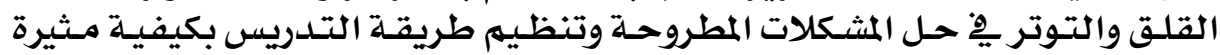
لكلانتباه والدافعية.

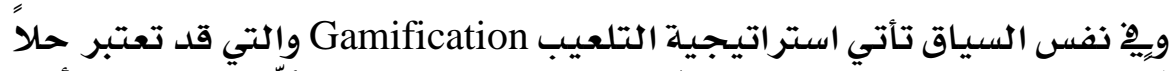

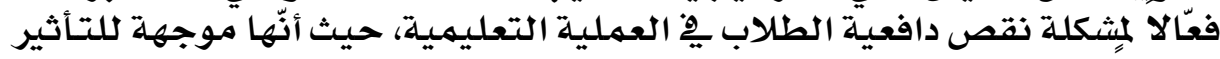

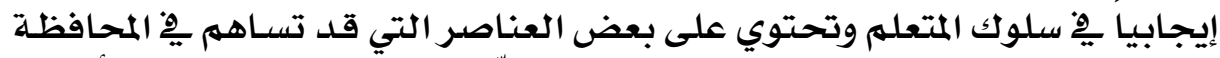

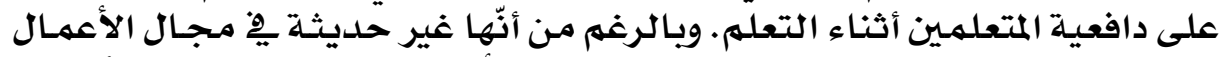

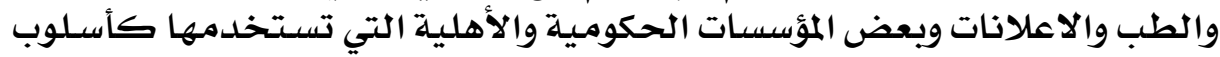

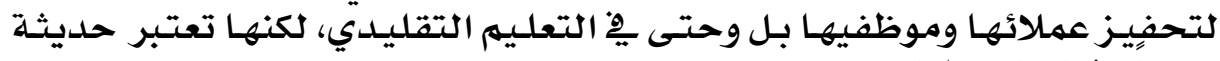

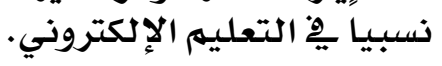

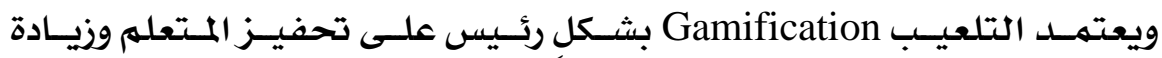

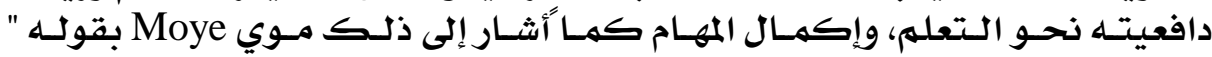

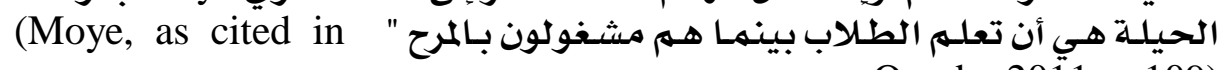
.Overby,20î1, p. 109)

ويعرف كاب استراتيجية التلعيب Gapp, 2012,p.10) Gamification بأنها

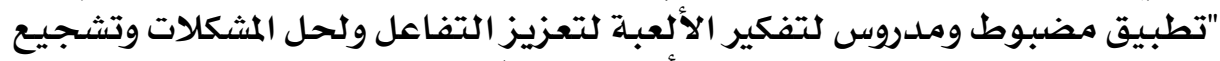

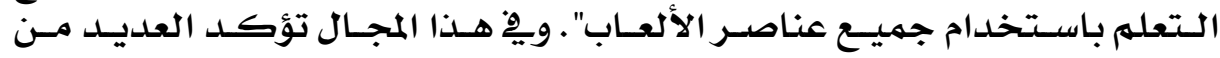

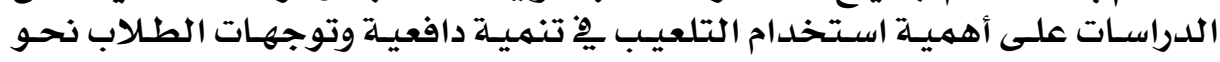
التعله.

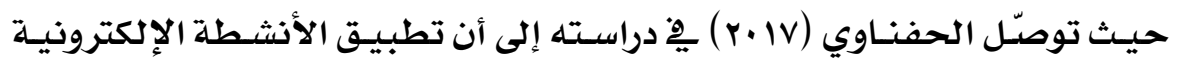

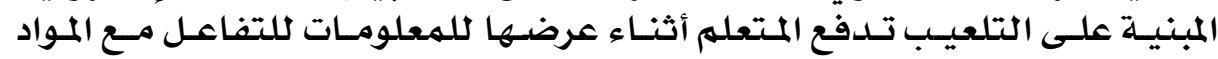




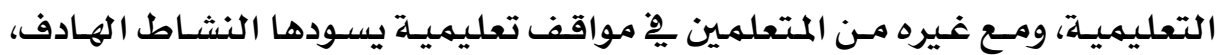
وتنهي مهارات التواصل واعله والتفاعل مـع البيئة المحيطة.

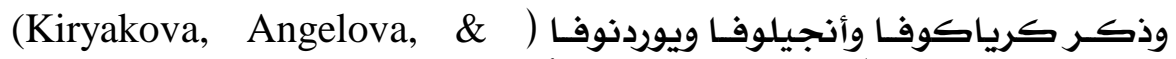

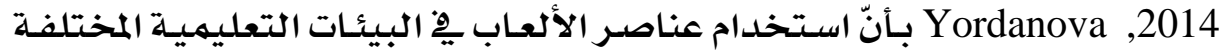

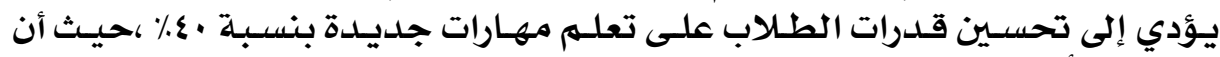

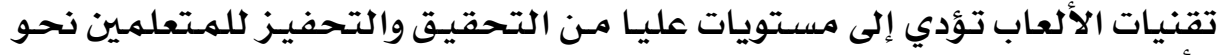
الأنشطة والمهام الواجب تودي القى مليهم أدائها.

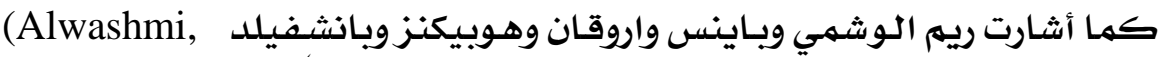
) بأن أهميـة التلعيب (Baines, ,Organ, ,Hopkins, \& Balanchedfield, 2014 تكمن يْ فهم قدرته على تغيير جزء من الطريقة التي يحدث بها التعله.

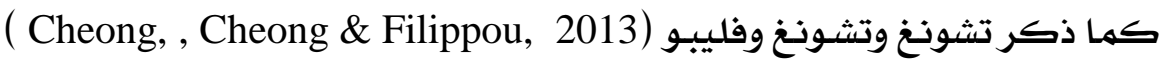

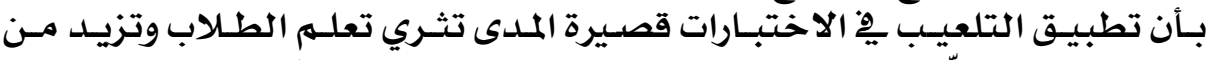

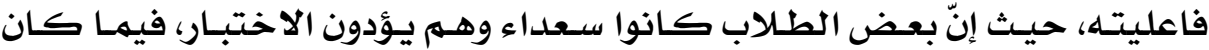
البعض الاخر يحاول بأقصى جهـ العهده لإكهماله.

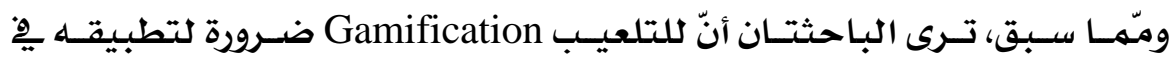

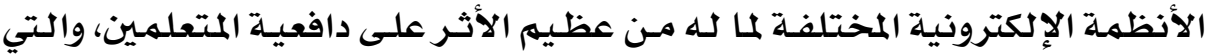

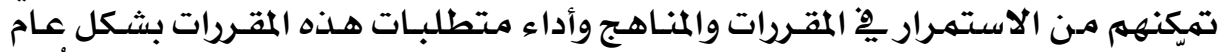

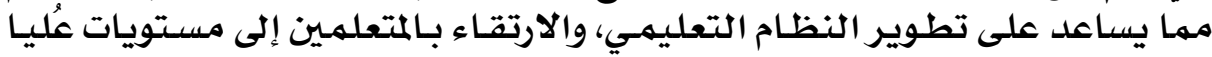
من التحفيزوالإنجاز.

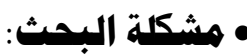

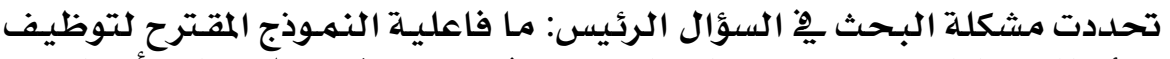

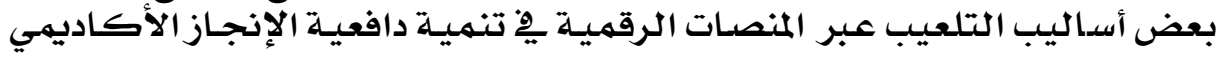

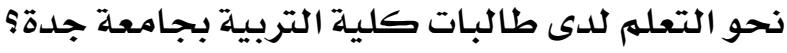

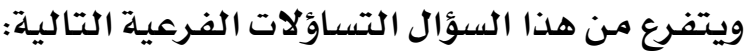

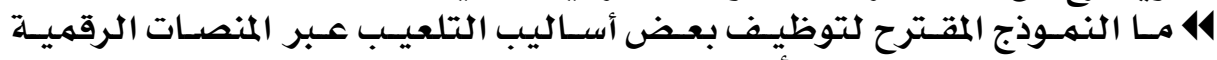

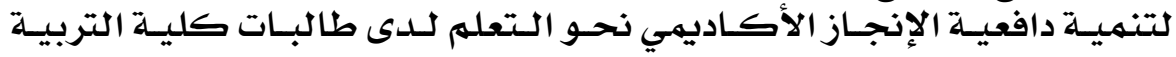

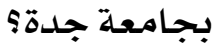

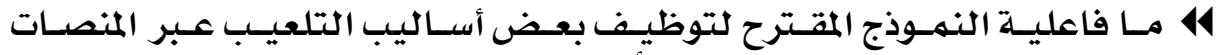

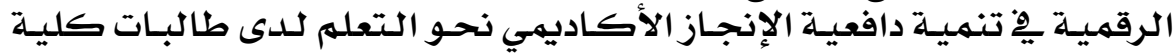

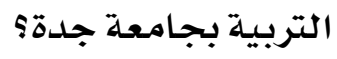

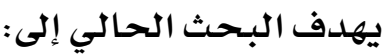

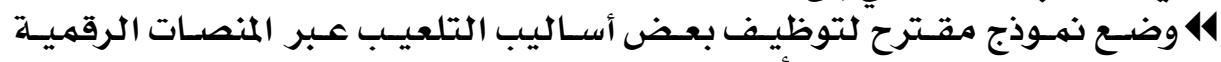

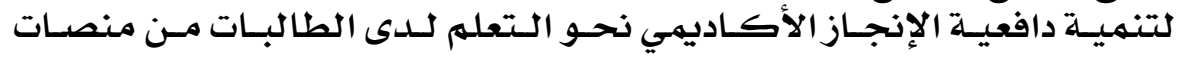

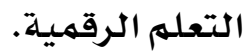




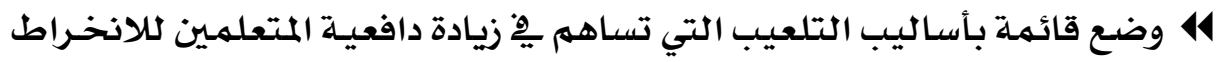

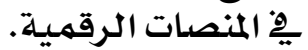

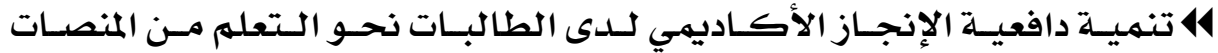
الرقمية.

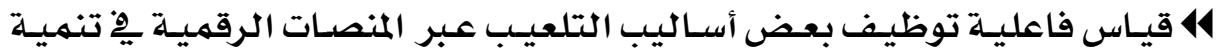

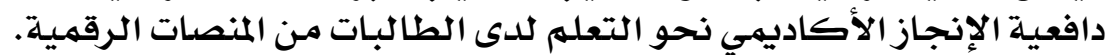

من المتوقع أن يستفيد من نتائج هذا البحث كالًا مـن:

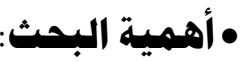

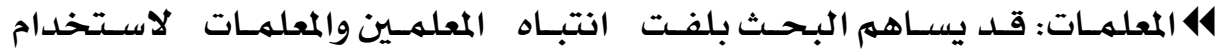

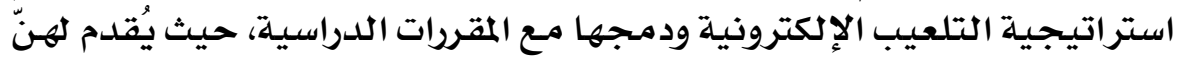

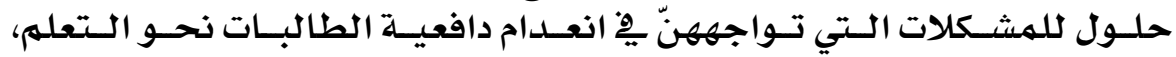

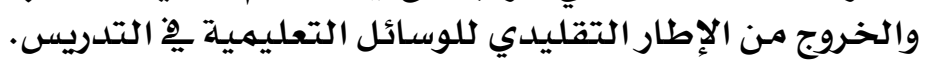

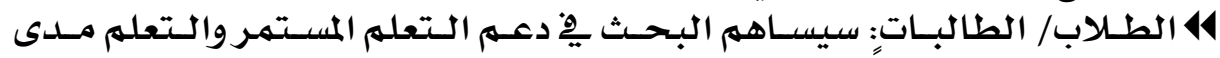

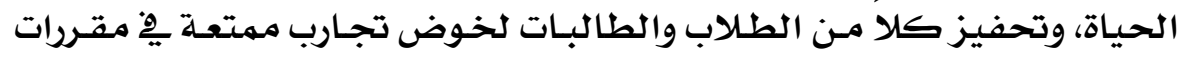

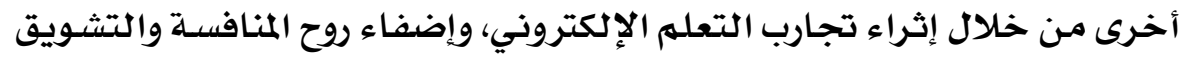

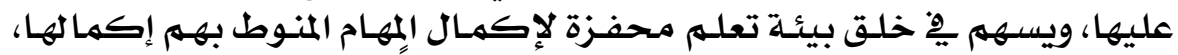

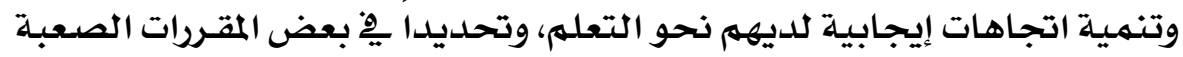

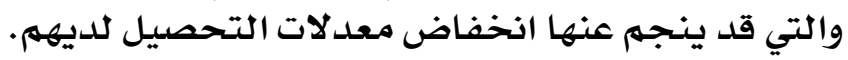

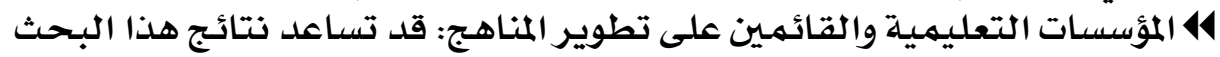

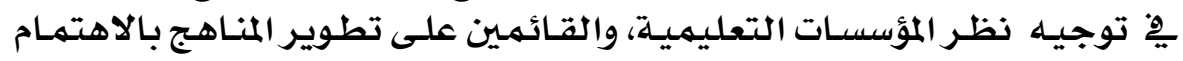

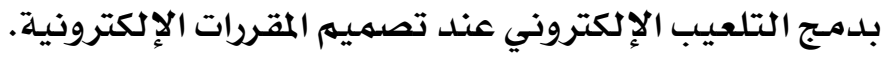

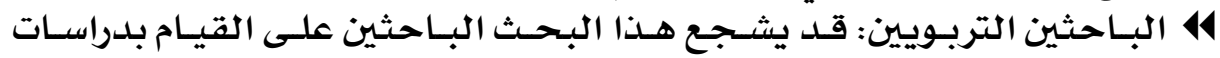

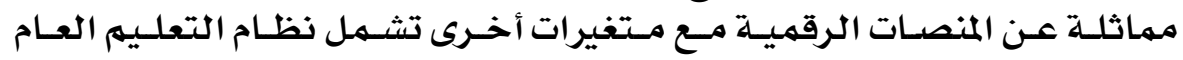

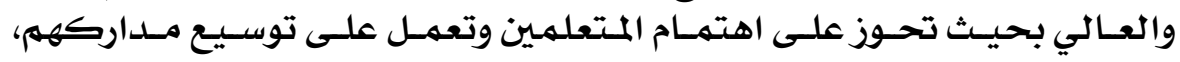

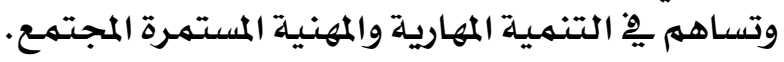

$$
\text { هدود البحث: }
$$

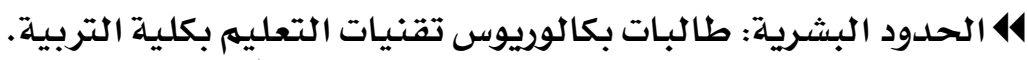

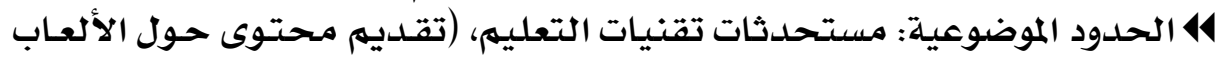

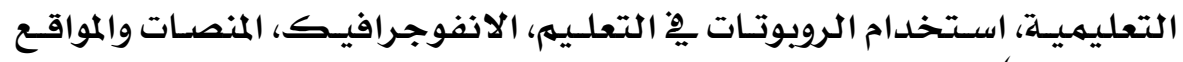

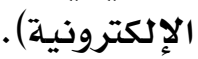

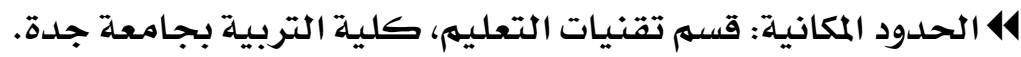

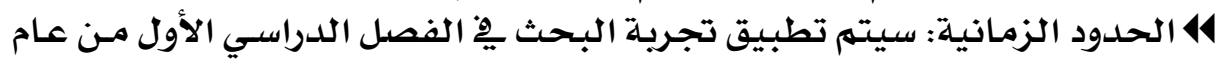

\section{$\boldsymbol{Y}$}




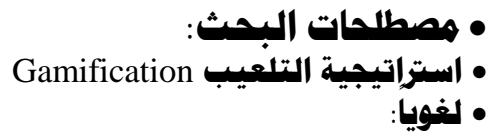

التلعيـب أو اللوعبـة Gamification، وهـو مصـطلح مشـتق مـن كلمـة Game

$$
\text { والتي تعني لعبة. }
$$

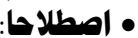

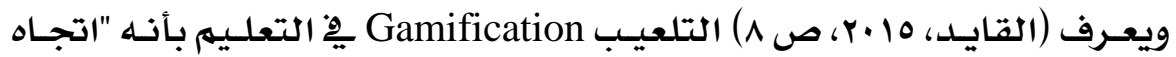

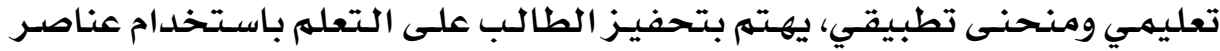

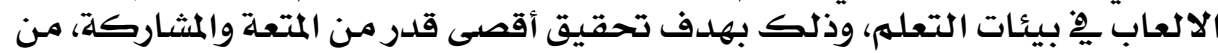
خلال جذب اهتمام المتعلمين لمواصلة التعلهم".

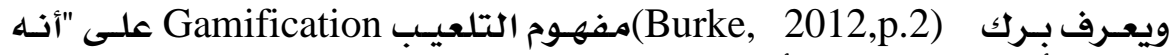

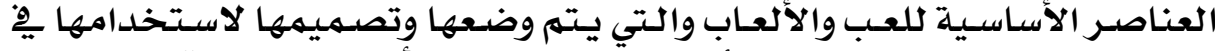

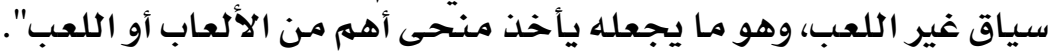
• تعريف (أساليب التلعيب عبر المنصات الرقيمية) إجرائياً:

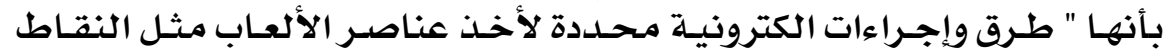

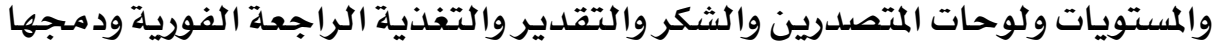

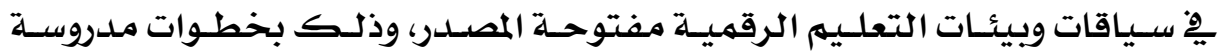

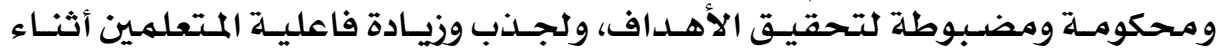

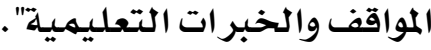

• المنصات التعليمية الرقتية (MOOCs):

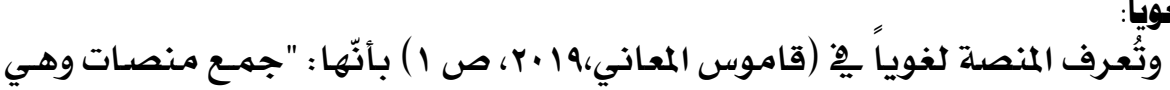

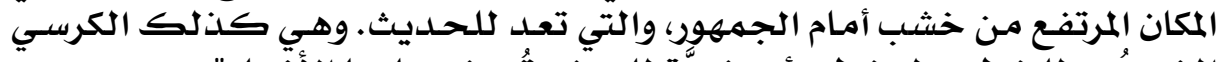

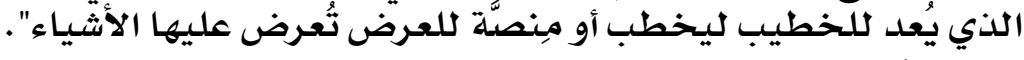

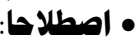

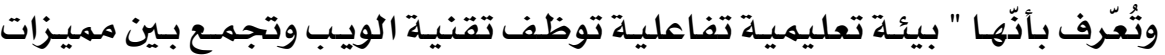

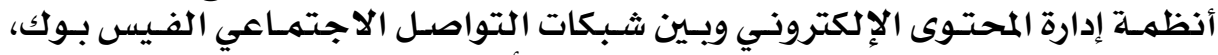

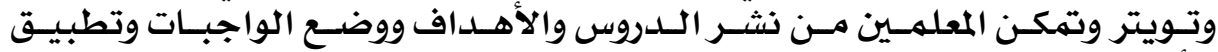

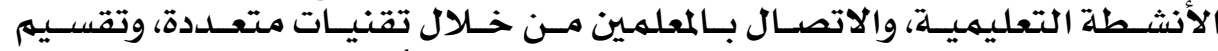

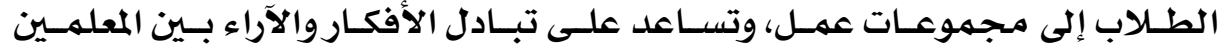

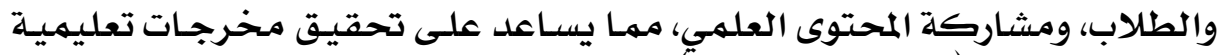

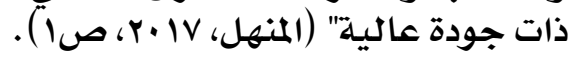

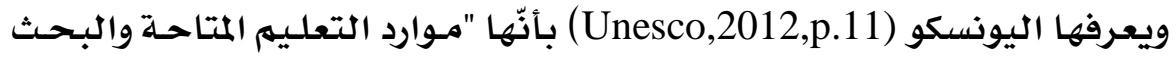

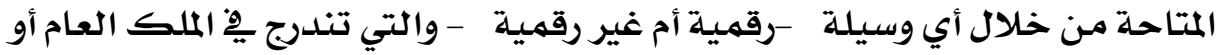

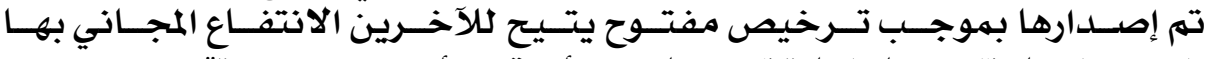

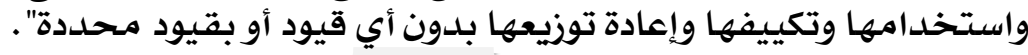




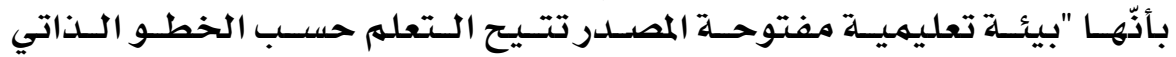

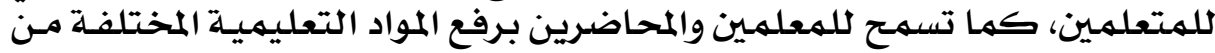

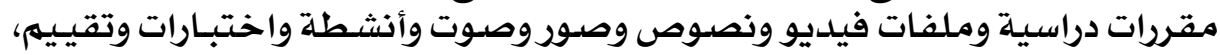

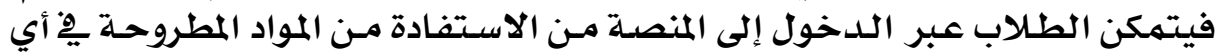

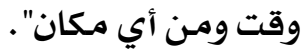

:Academic achievement motivation

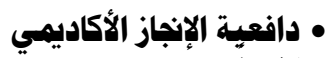

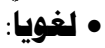

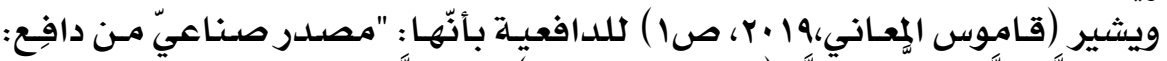

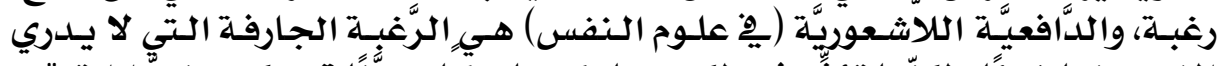

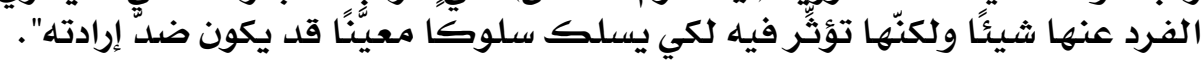
• اصطلاحا:

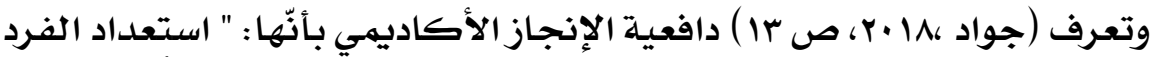

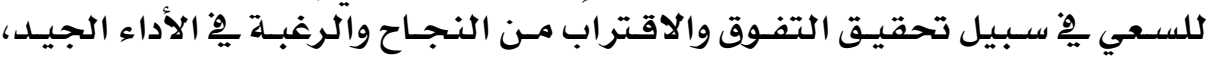

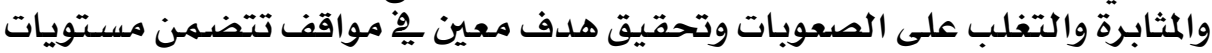

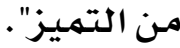

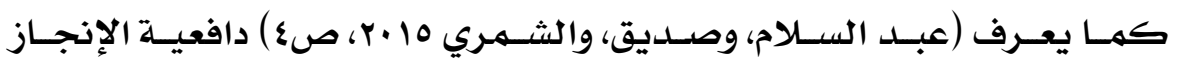
الأكاديهي بالتعريف التالي:

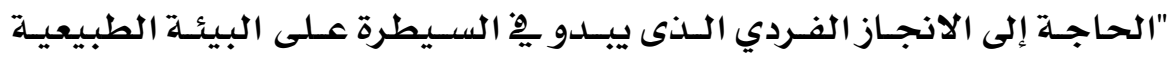

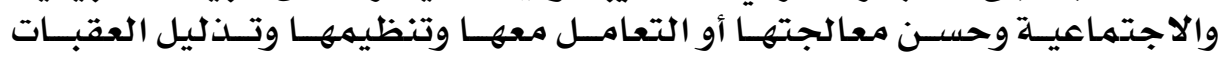

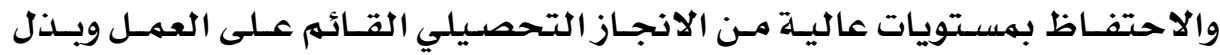

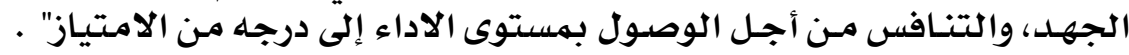

• تعريف دافعية الإنجاز الأكاديمي إجرائيا:

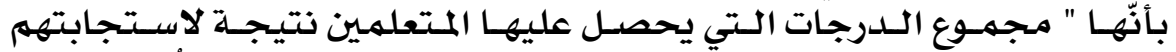

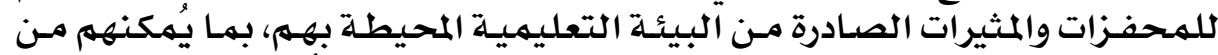

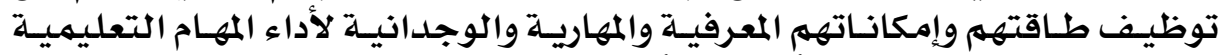

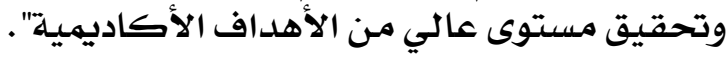

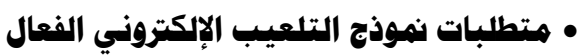

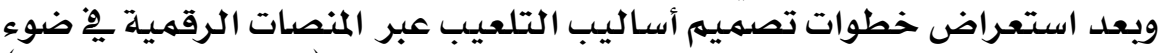

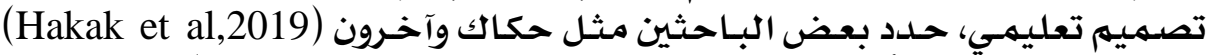

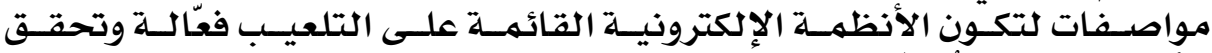

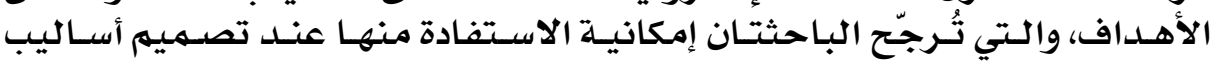
التعليب، كالتآي: الأي

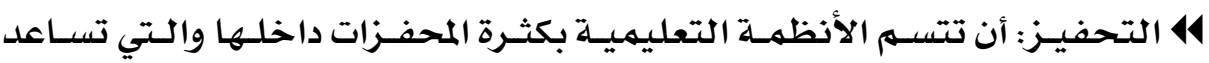
المتعلمـين على الاستمـرار فِ التعلهم.

\section{YY}




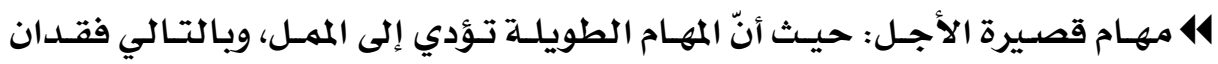

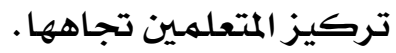

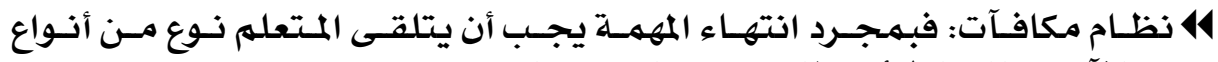

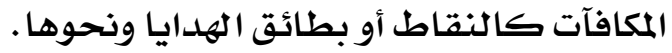

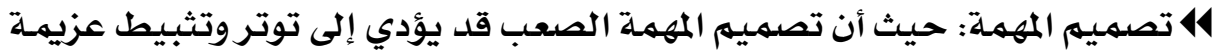

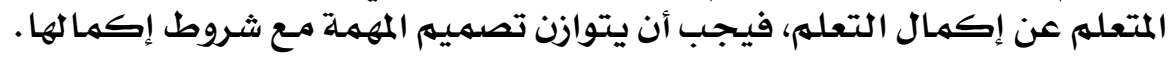

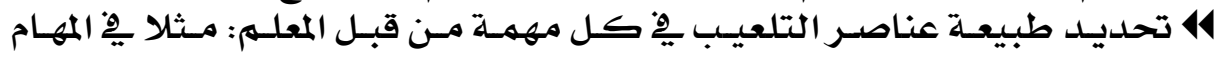

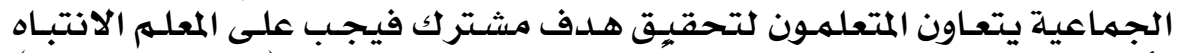

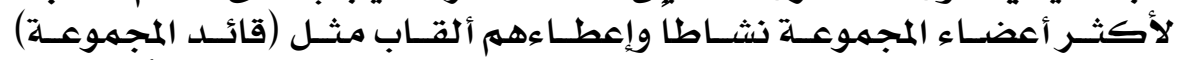
كمكافئة وهكنا، وهذا يساعد الطولاب على معرفة تكنيك تحقيق الأهداف فِ

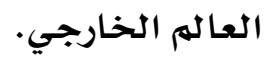

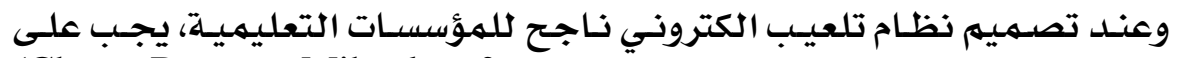

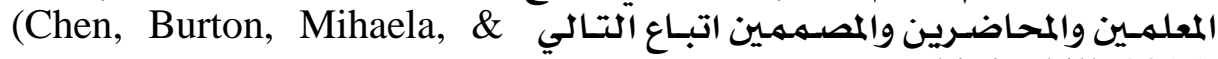
:Whittinghill, 2015)

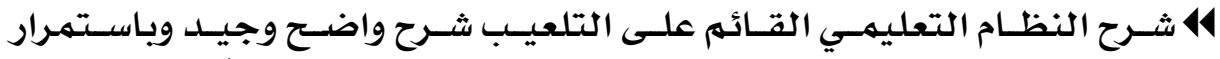

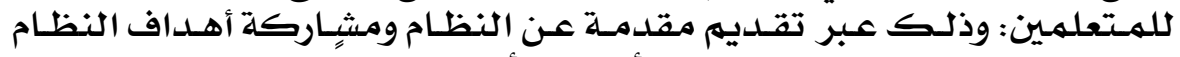

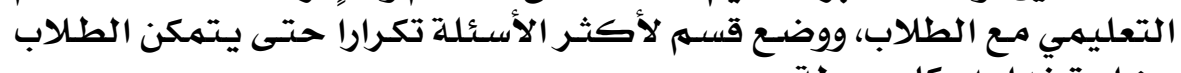

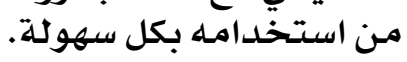
414 التحكم بصعوبة النظام التعليمي القائم على التلعيب: وذلك بتلو بتوضيح خاصية

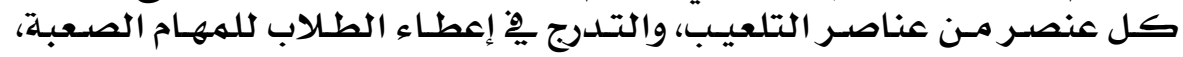

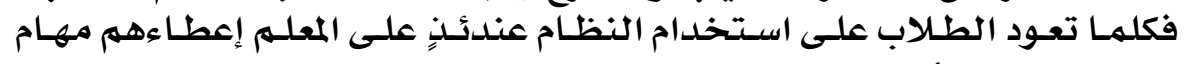

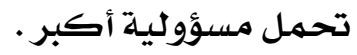

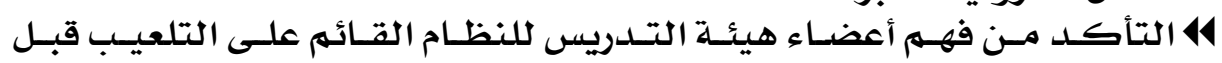

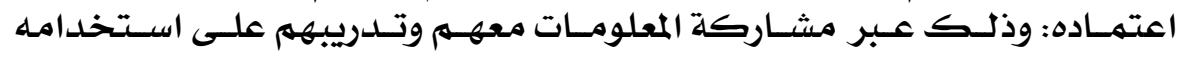

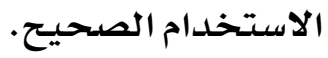

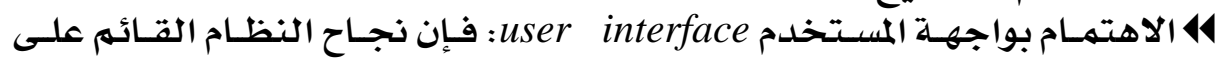

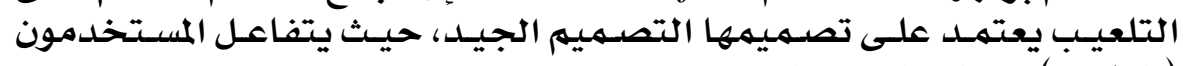
(الطلاب)" معهها مباشرة وباستمرار.

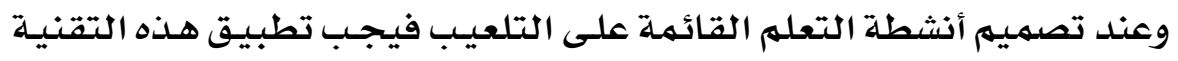

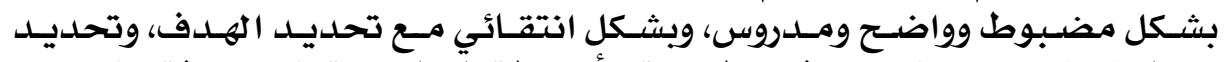

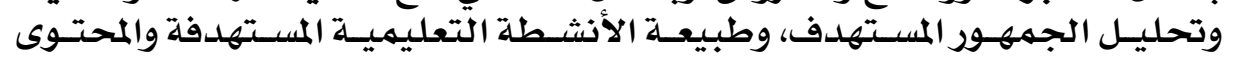

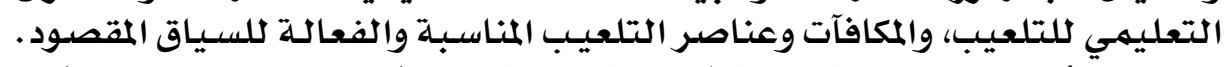

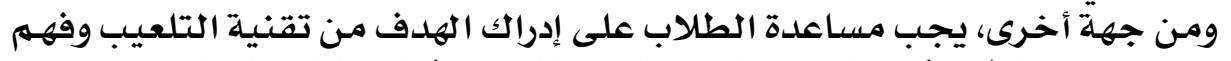

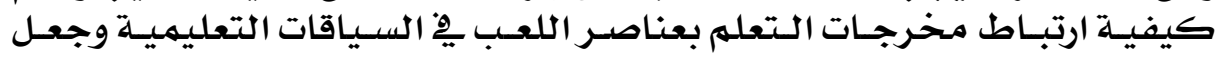

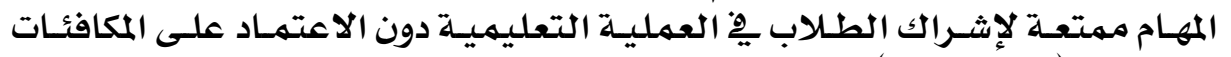

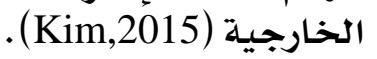

\section{Mr}




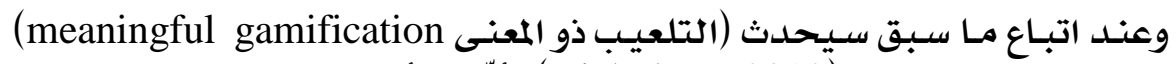

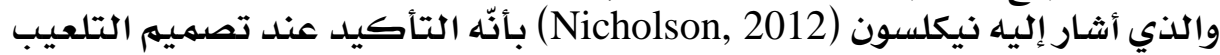

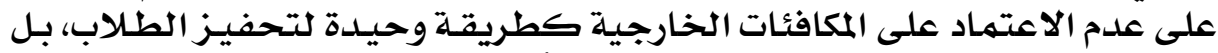

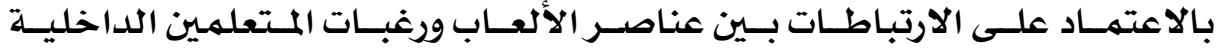
وأهدافهم.

\section{• التصميم الأولي لنهوذج التلعيب المتزخح}

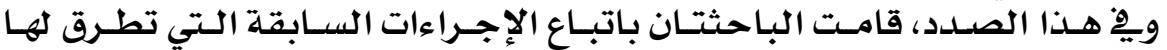

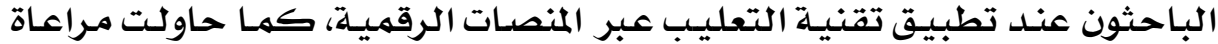

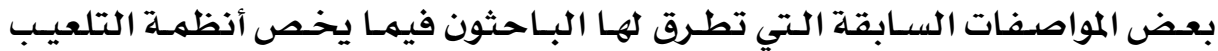

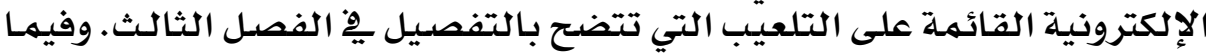

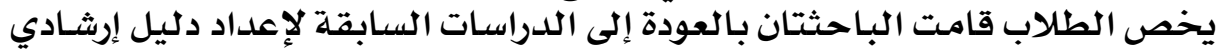

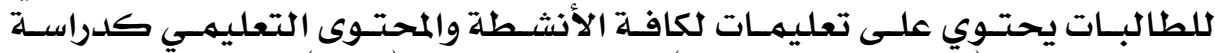

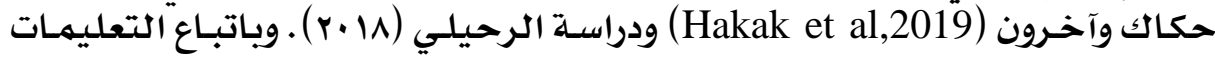

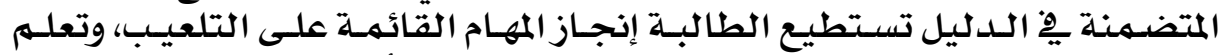

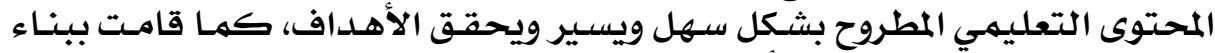

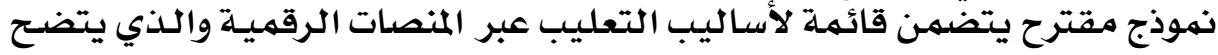
بالتفصيل ِِّ الفصل الثالث وهنا استعراض للنقاط النقاط الرئيسية، كالتالي: • الصساب الشخصي (personal profile): قامـت الباحثتان بترجمهـة ديناميكِيـة التعبير عن الـذات لتتمثل هِّ ميكانيكيـة

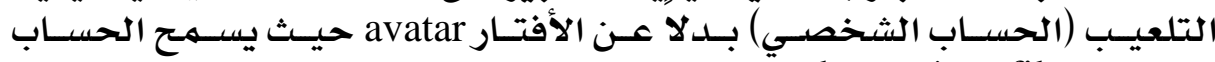

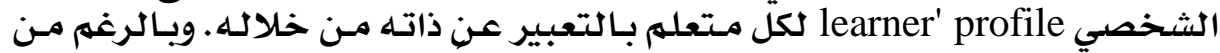

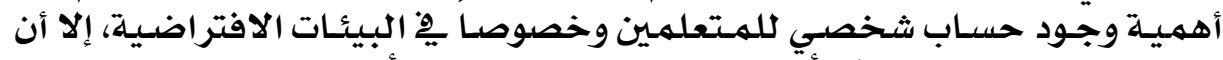

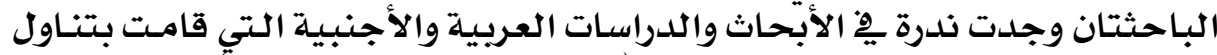

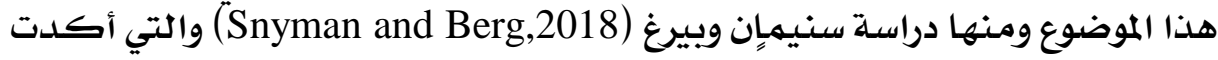

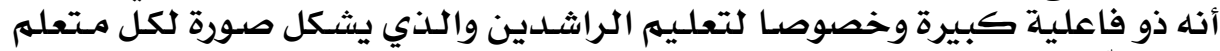

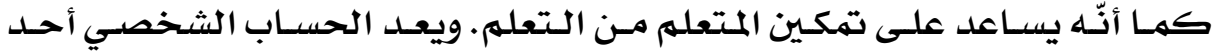

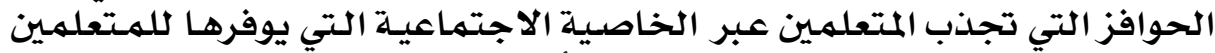

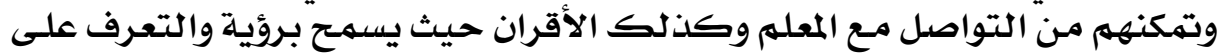

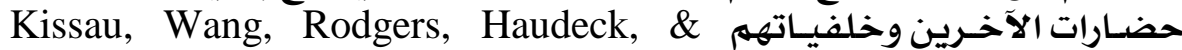
.(Biebricher, 2019) • الفطو الذاتي points:

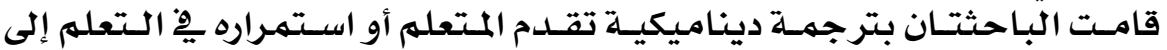

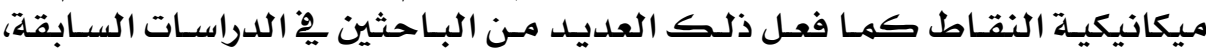

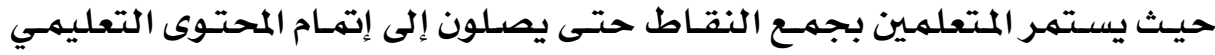

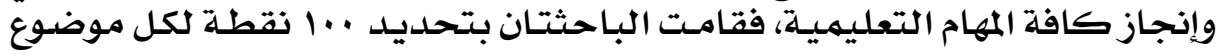

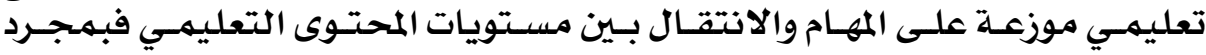

\section{r $\varepsilon$}




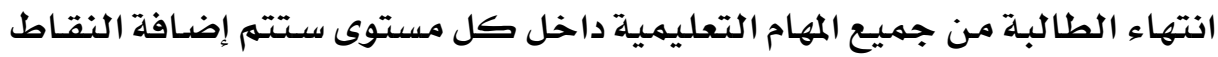

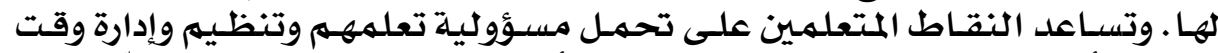

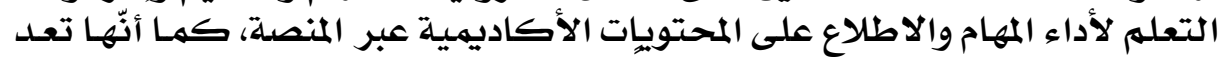

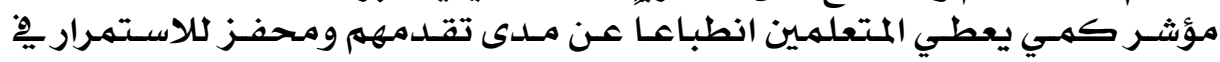

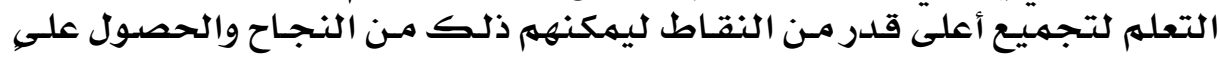

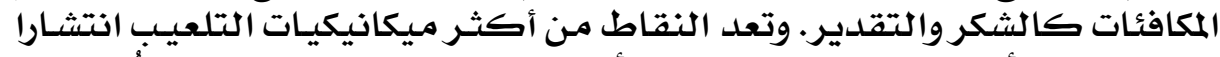

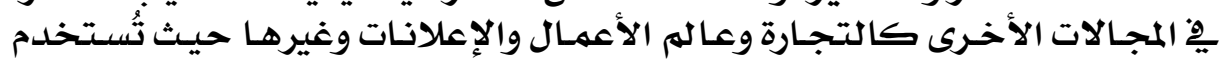

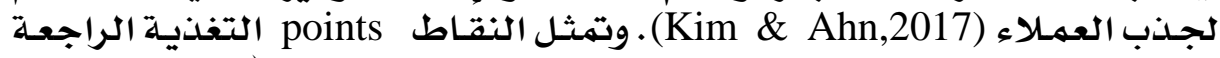

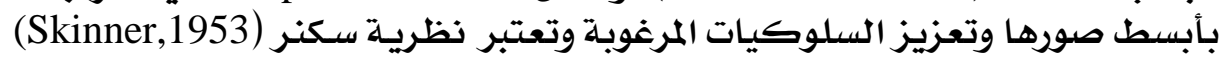

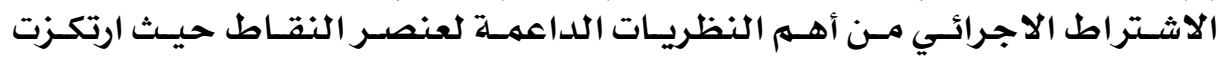

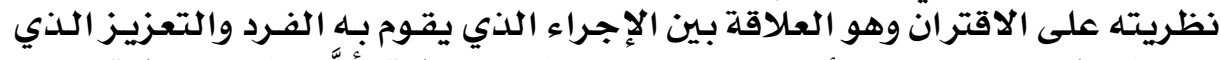

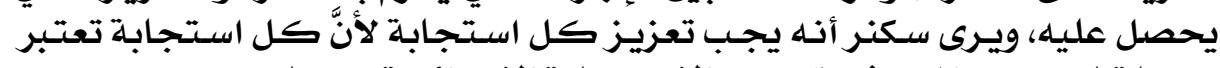

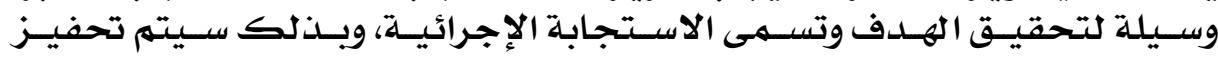

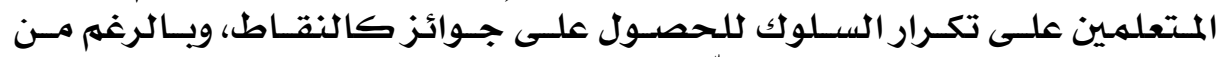

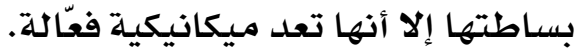

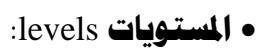

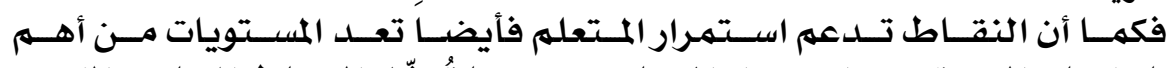

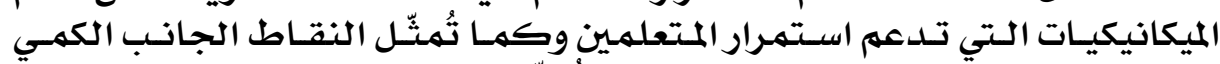

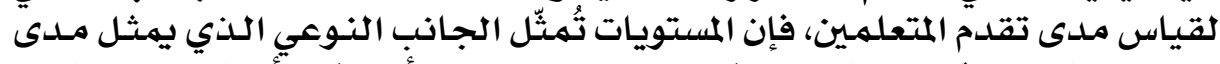

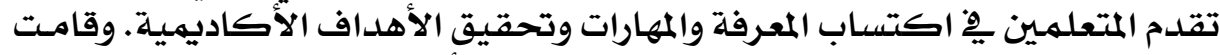

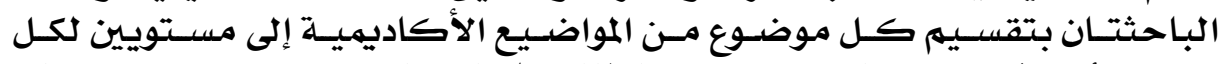

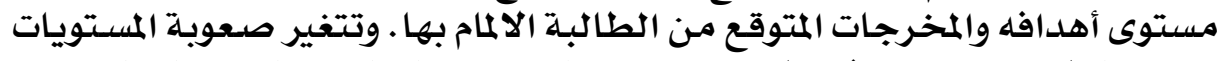

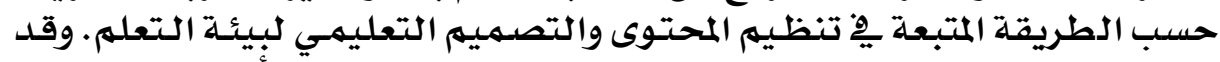

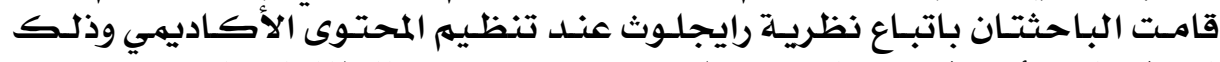

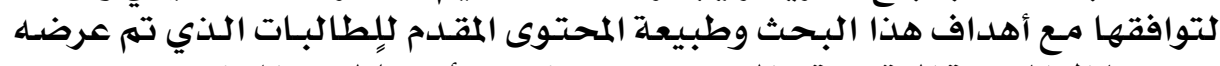

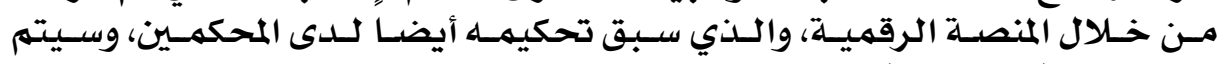

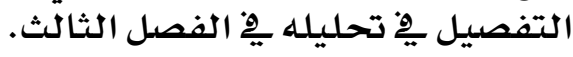

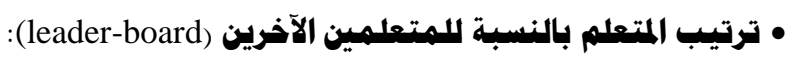

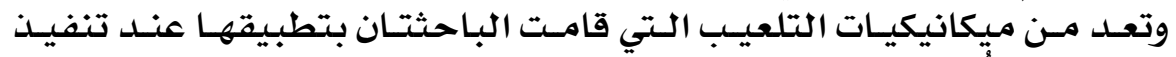

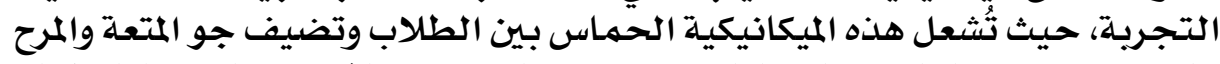

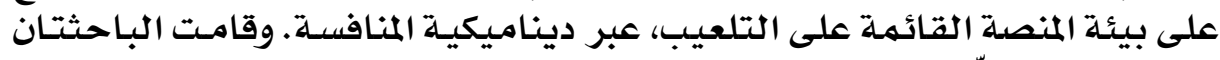

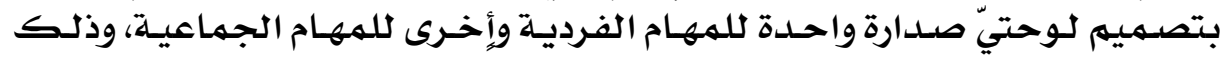

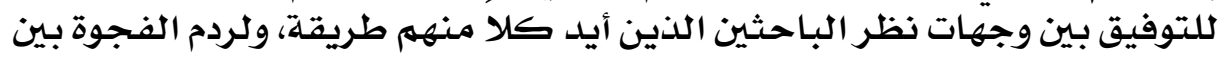

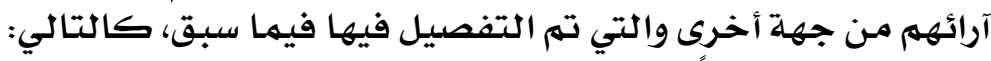
• ترتيب الطالبة مقارنةً برزهيلاتها الأخريات:

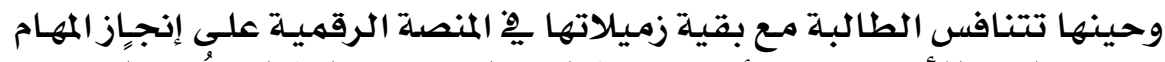

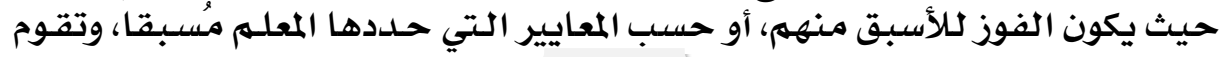

\section{$\boldsymbol{r} \theta$}




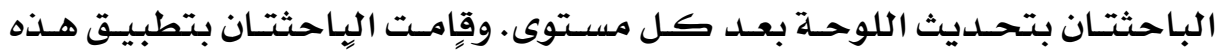

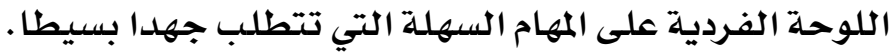

• ترتيب المبمهوعة هقارنةً بالمبموعات الأخرى:

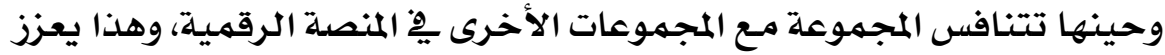

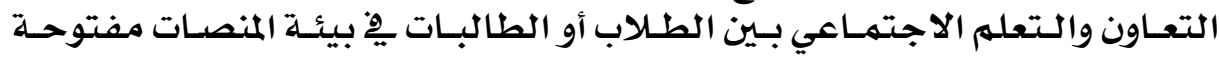

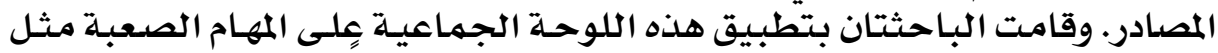

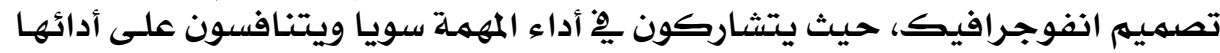

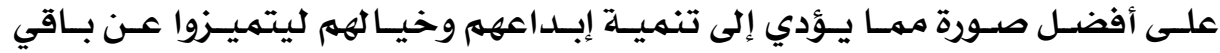

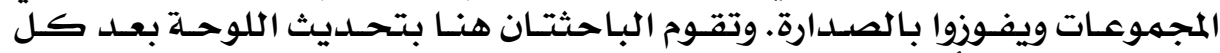

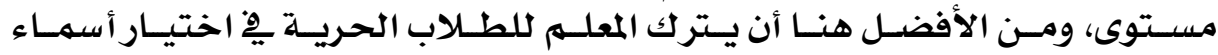

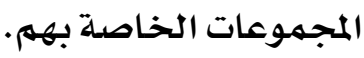

• المهام التعليمية والتي تُمثل التمديات (challenges):

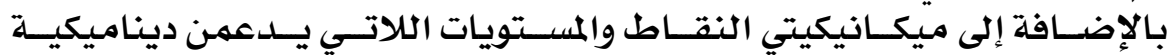

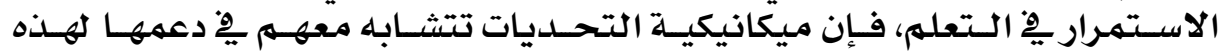

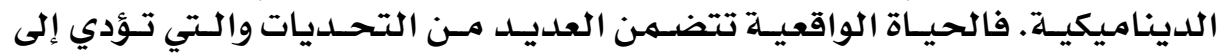

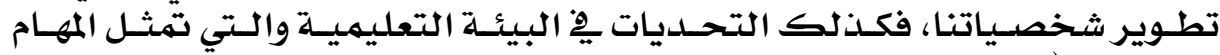

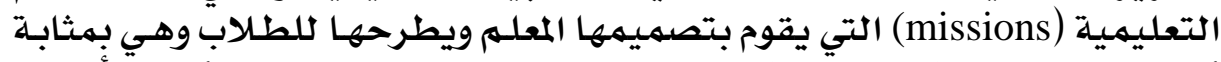

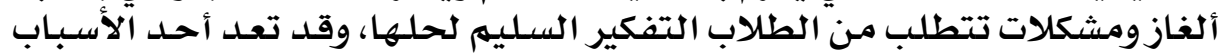

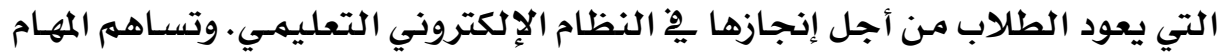

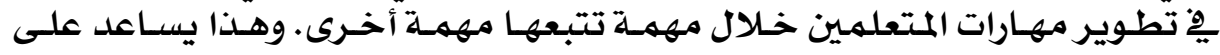

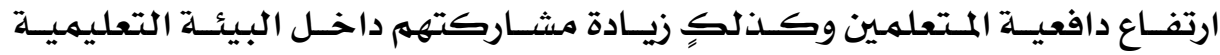

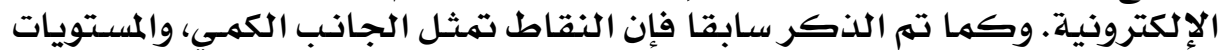

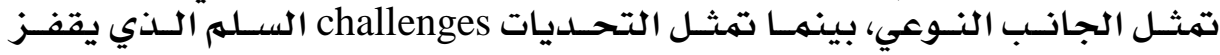

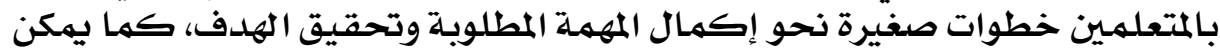

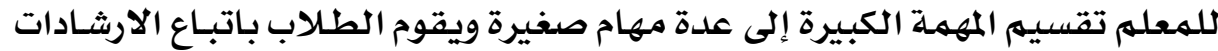

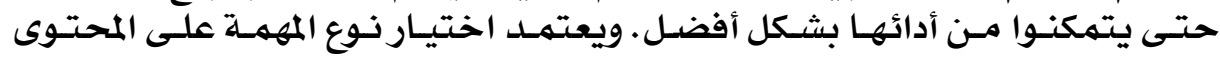

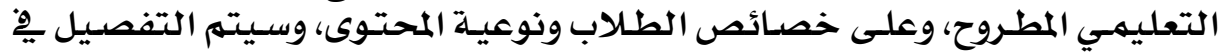

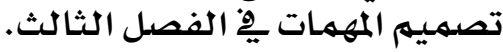

• المكافآت التي يمصل عليها المعا المتعلم (rewards):

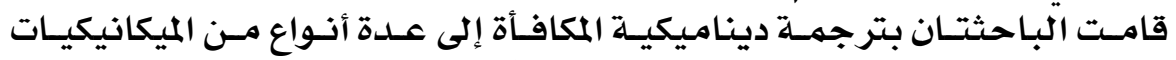

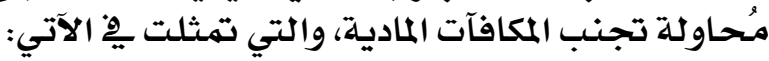

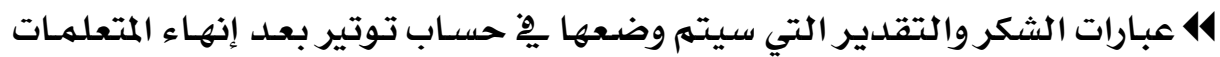

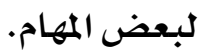
\ نشر أفضل مشروع قامت الطالبـات بإنشائه عبر تويتر، وتنويع طـرق النشـر فهـرة

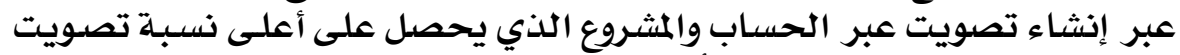

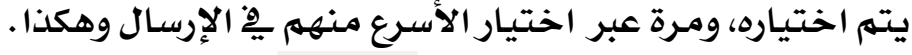

\section{ry}




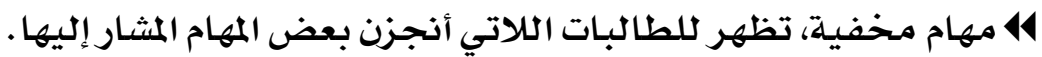

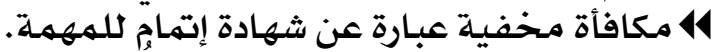

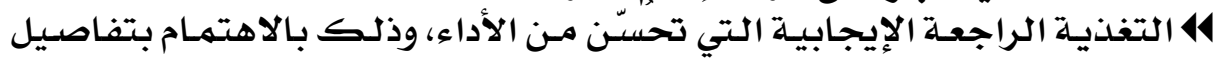
المهمة.

• استخدام تطبيقات الشبكة الاجتهاعية هـ أساليب التلهيب:

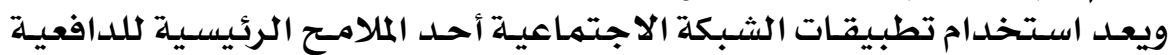

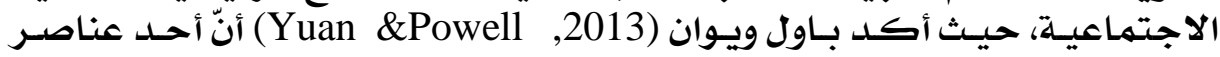

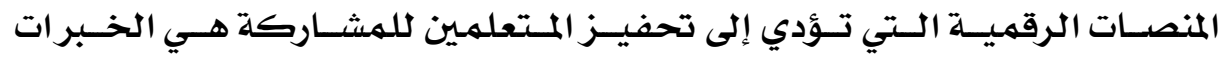

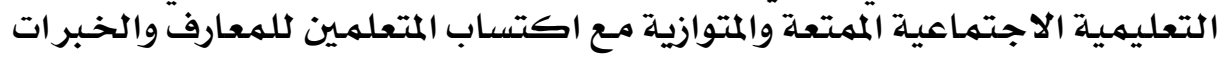

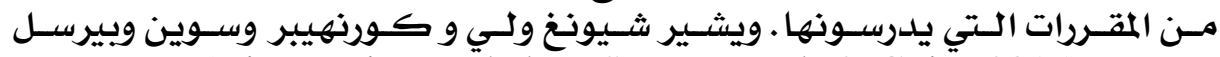

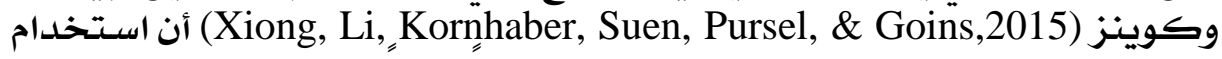

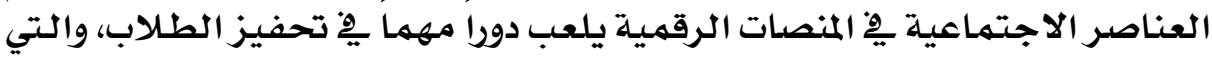

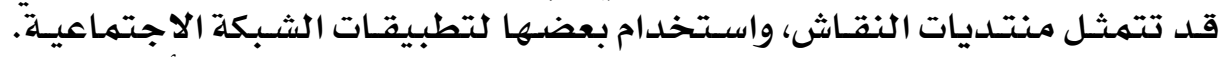

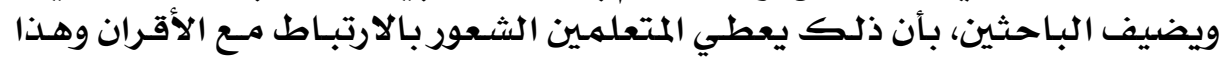

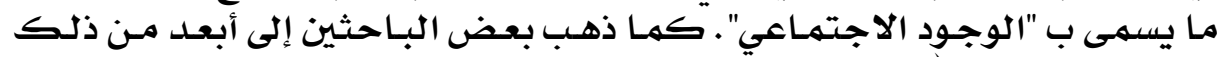

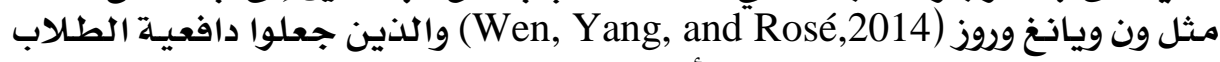

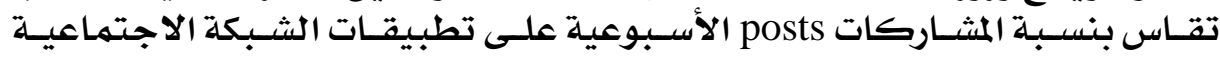

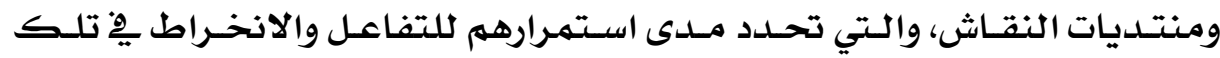
المقررات.

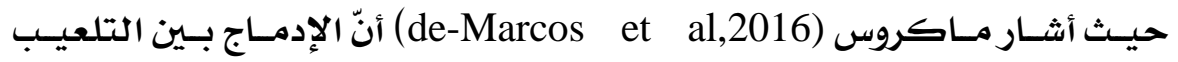

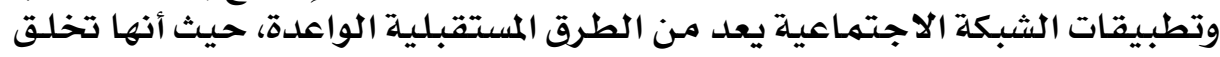

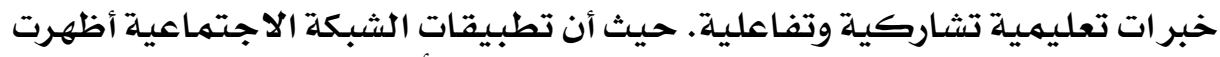

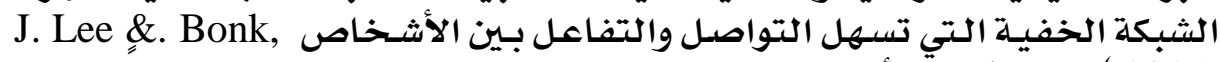

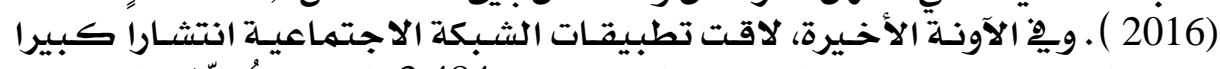

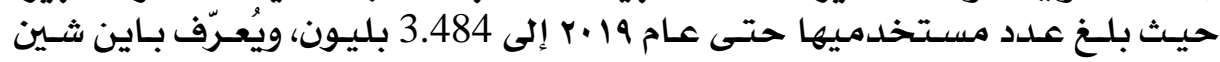

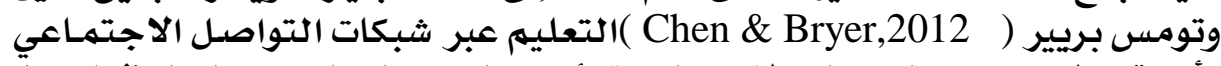

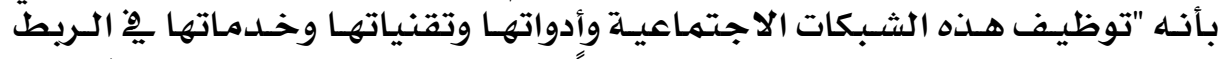

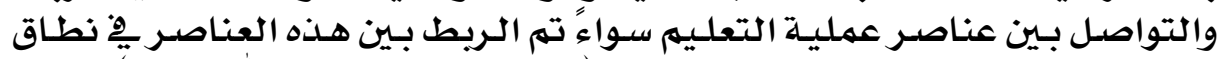

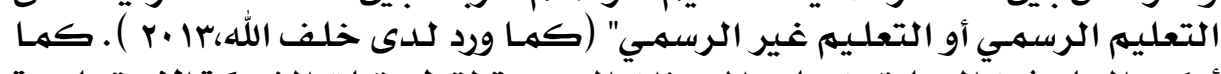

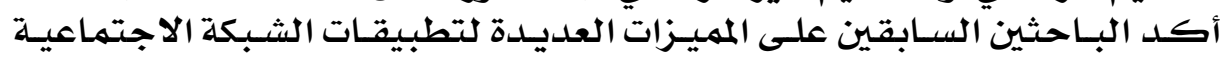

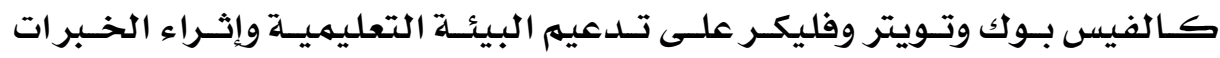
التعليمية.

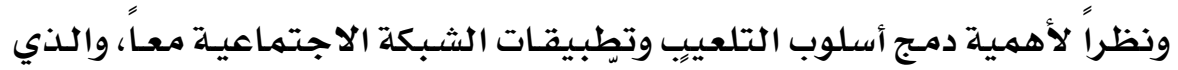

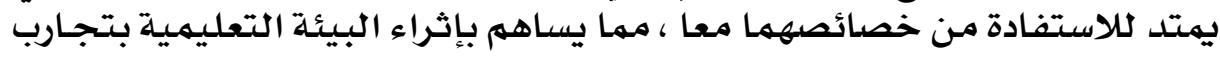

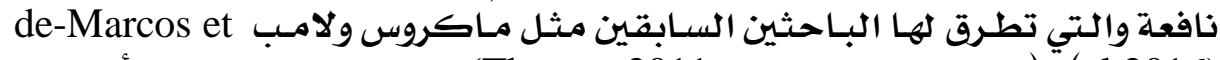

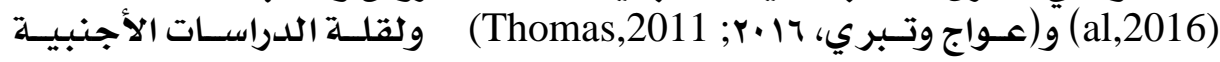




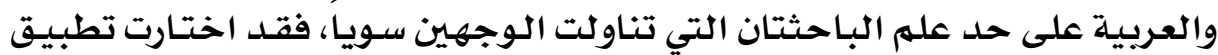

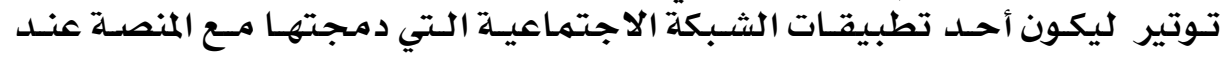

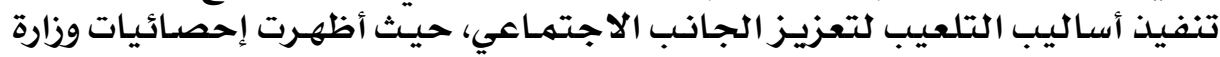

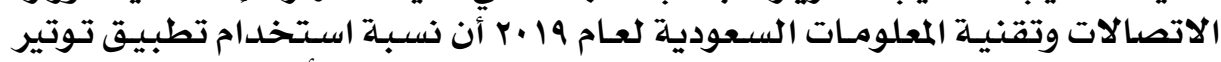

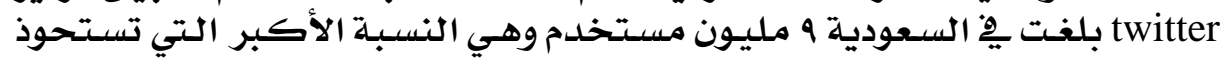

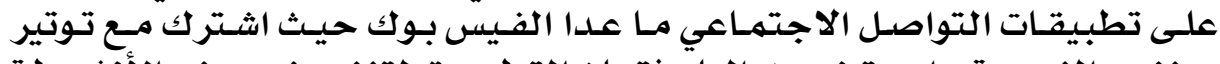

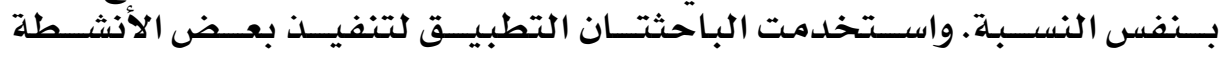

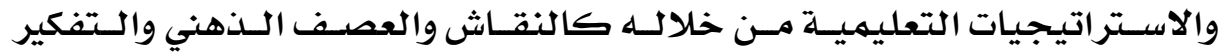

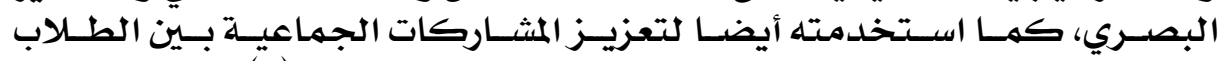

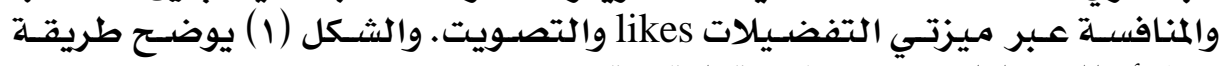
عمل أساليب التلعيب عبرتي المنفي المنصة الرقهية:

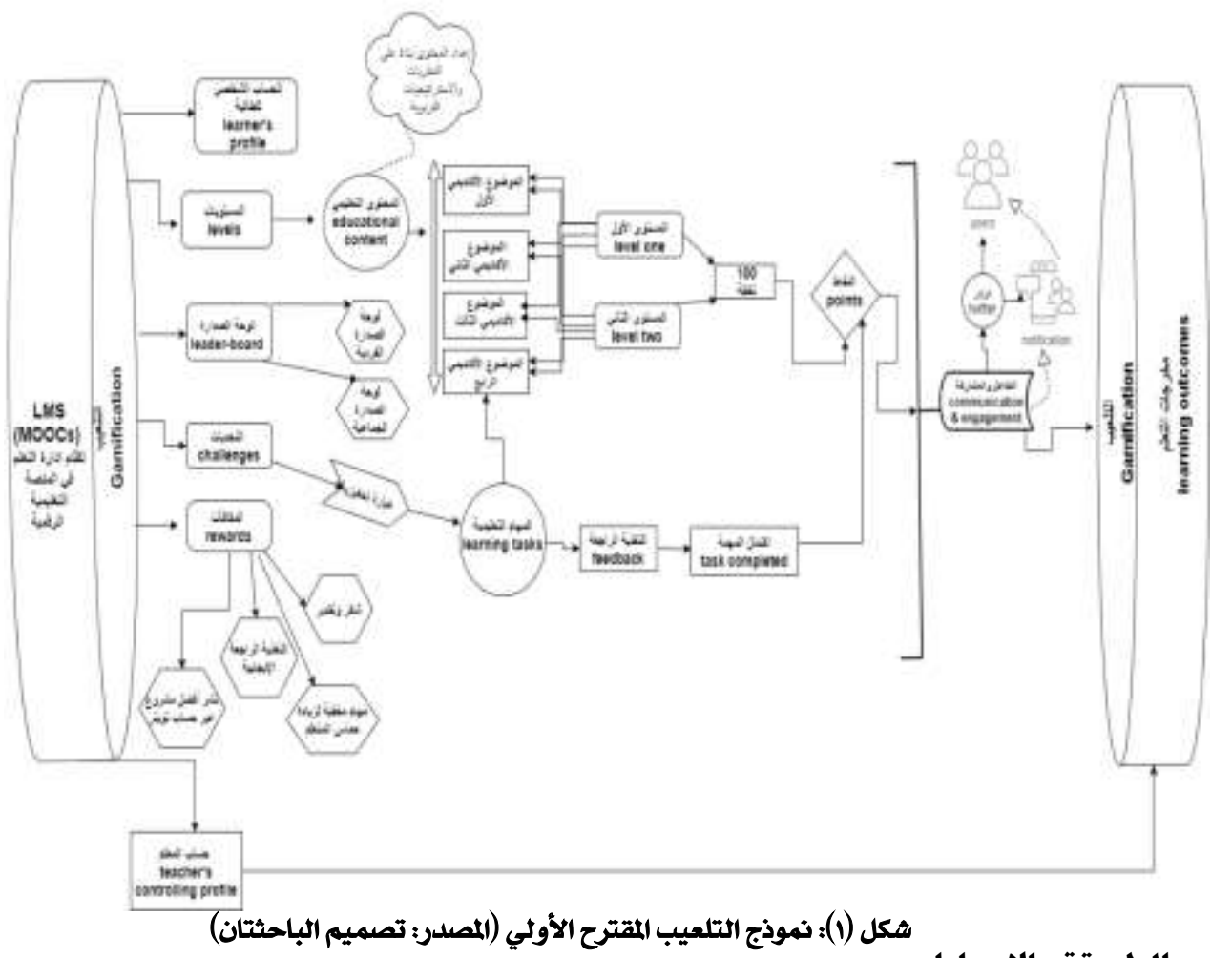

$$
\text { • • هنهجية البحة والإجراءات: }
$$

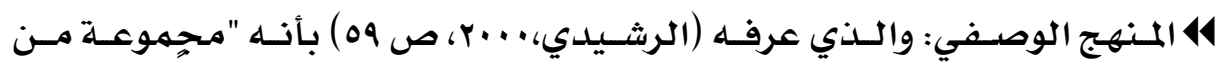

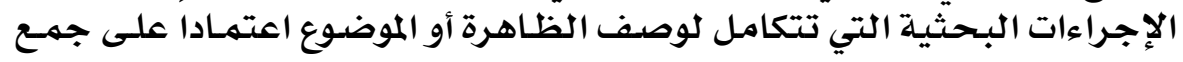

\section{rᄉ}




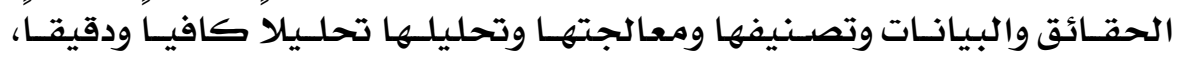

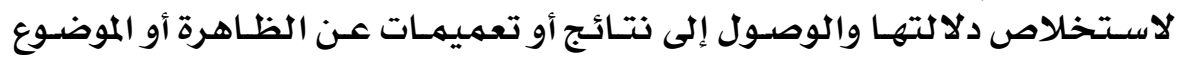

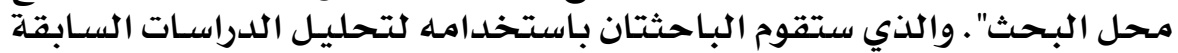

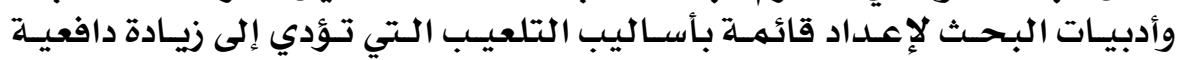

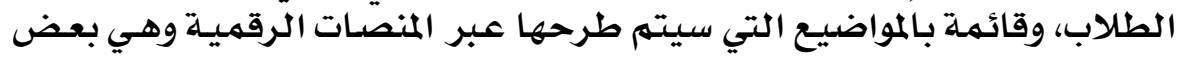

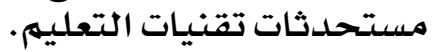

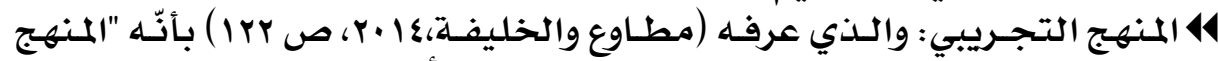

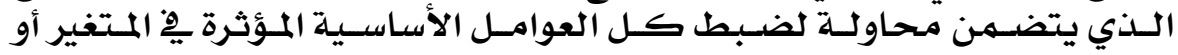

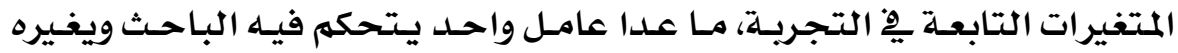

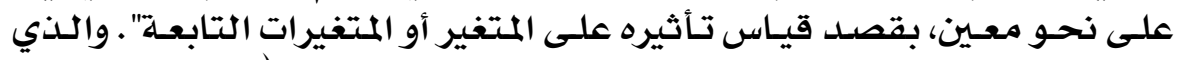

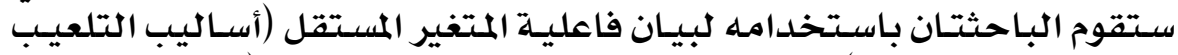

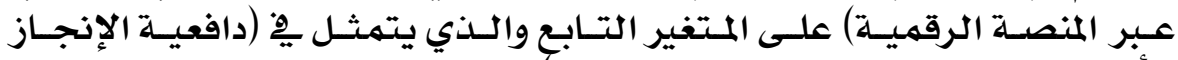
الأكاديهي نحو التعلهم من المنصدة المسئفير الرقمية).

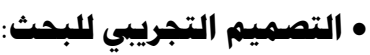

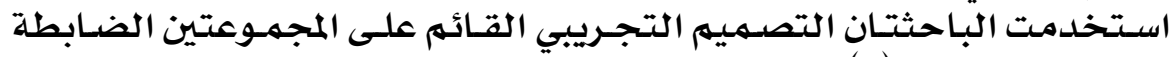

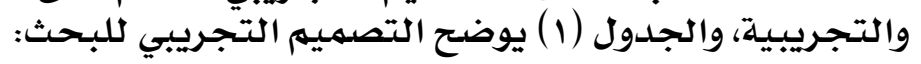

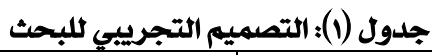

\begin{tabular}{|c|c|c|c|}
\hline القياس البعلى & المعالجت التجريييت & القياس القبلى & المجموعت \\
\hline 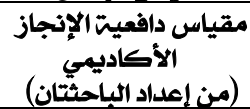 & التدريس باستخدام المنصتي & 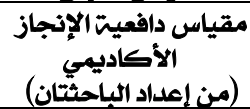 & مجموعت ضابطت \\
\hline 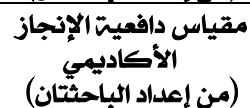 & التدريس باستخدلام أساليب التبليب & 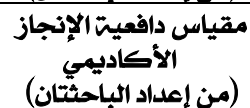 & مجموعت تجريبيت \\
\hline
\end{tabular}

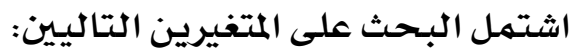

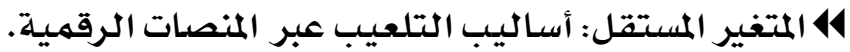

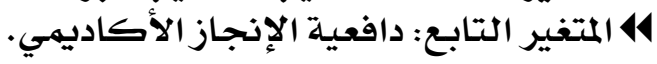

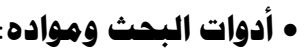
تضهن البحث إعداد المواد والأدوات التتالية:

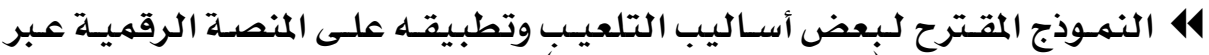

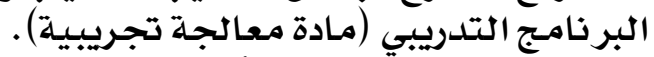

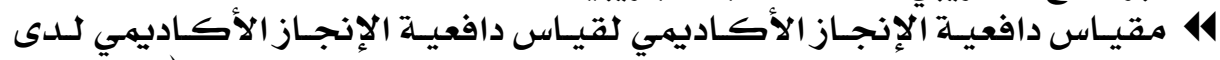

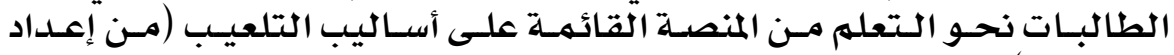

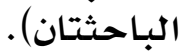

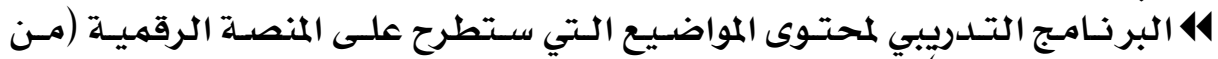

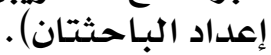
41 الدليل الإرشادي للطالبات (من إعداد الباحثتان). 


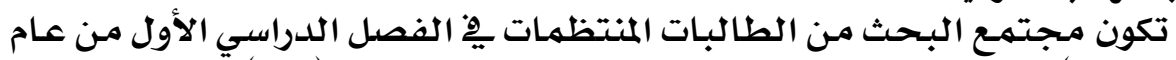

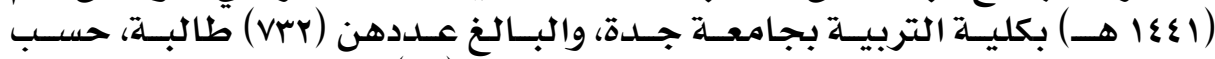

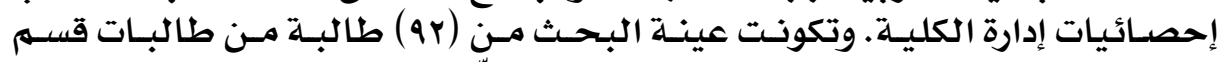

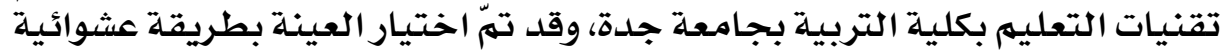

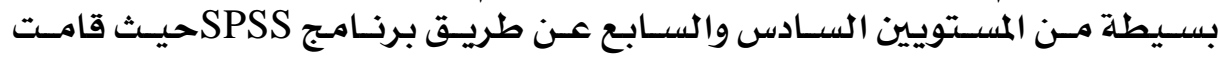

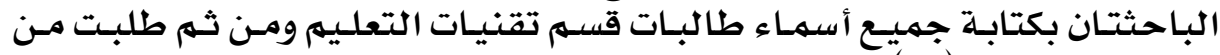

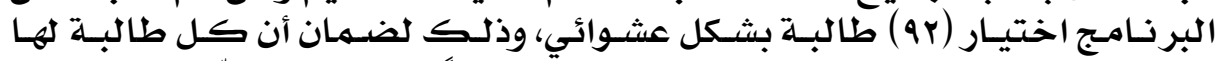

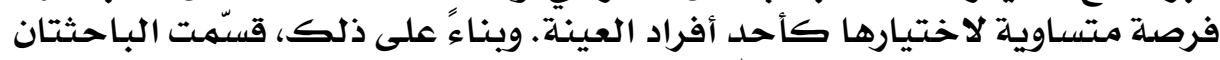

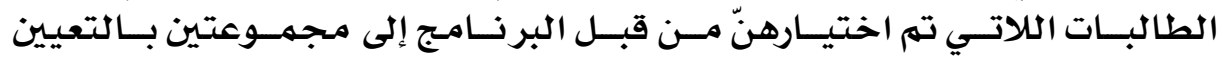

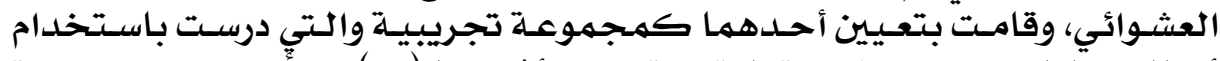

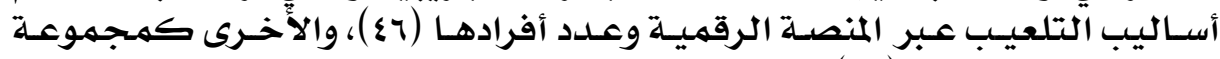

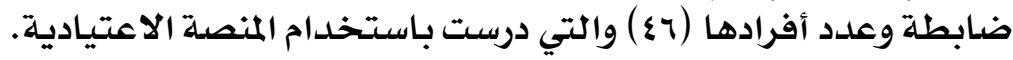

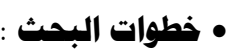

لتحقيق أهداف البحث، والإجابة البحابة على أسئلته واختبار فروضه، اتبعت الباحثتان

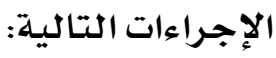

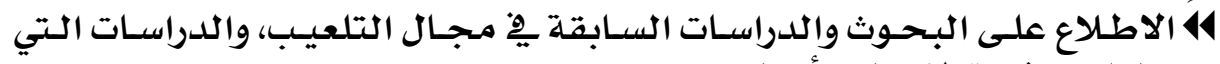
تناولت دافعية الإنجاز الأكادئيسي.

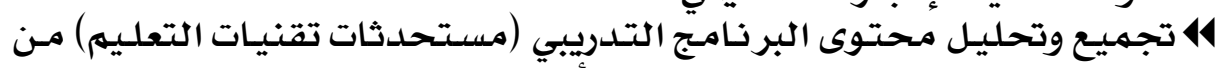

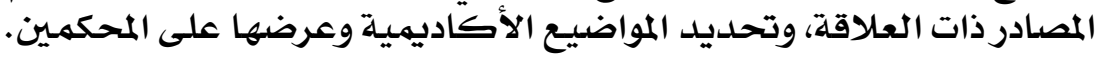

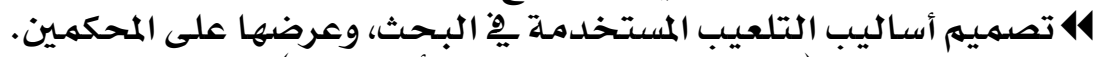

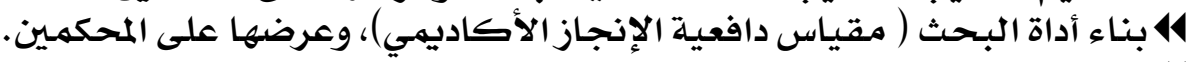

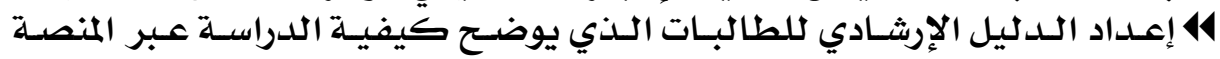

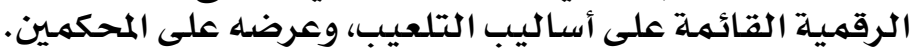

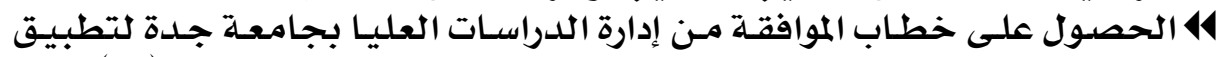

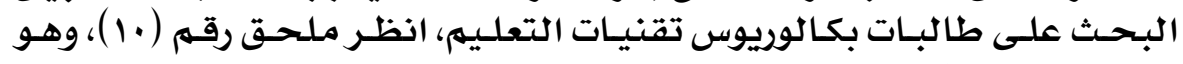

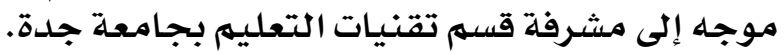

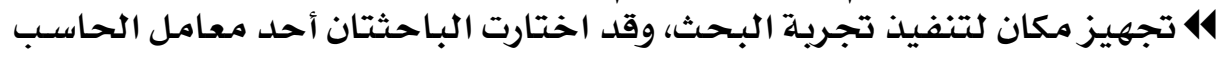

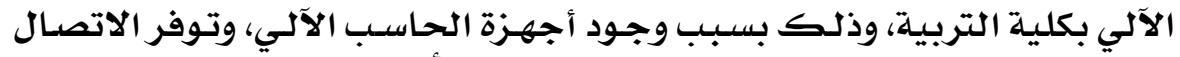

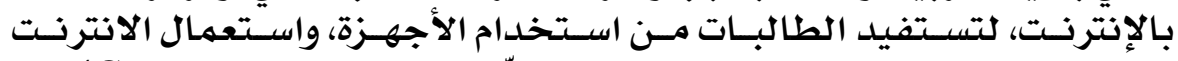

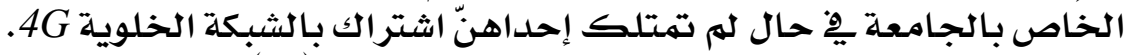

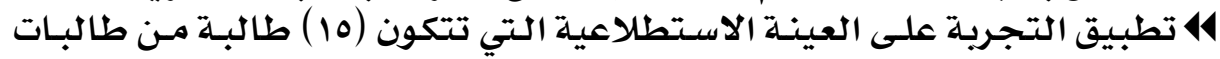

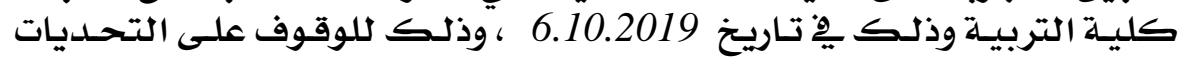

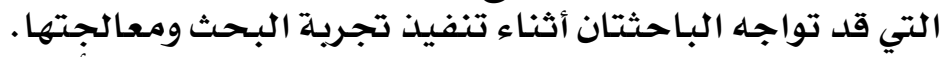

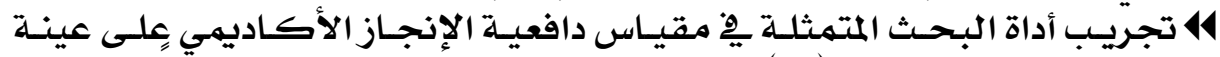

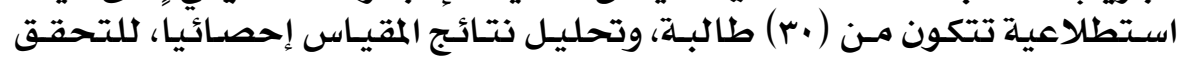
من صدق وثبات المقياس.

\section{$\varepsilon$}




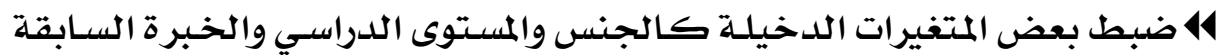

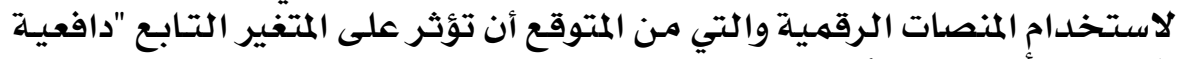

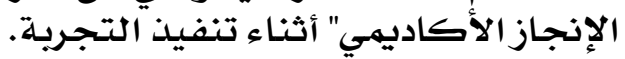

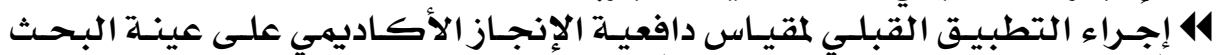

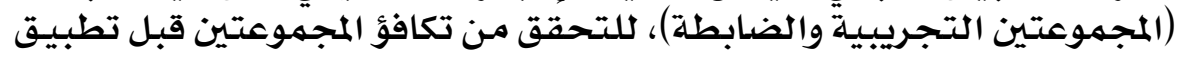
التجربة.

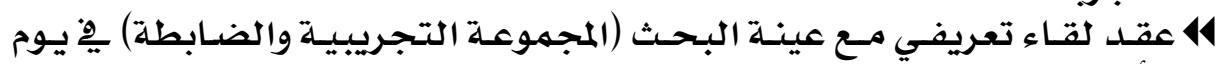

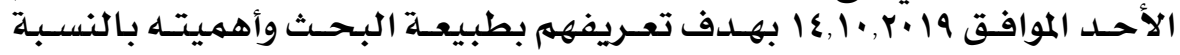

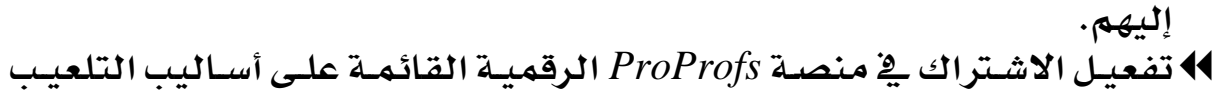

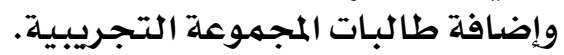

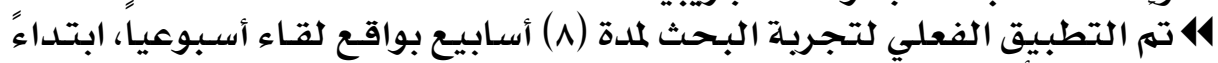

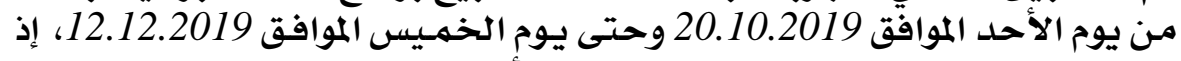

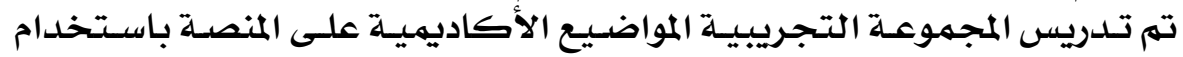

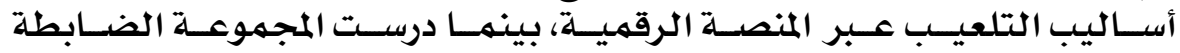

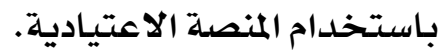

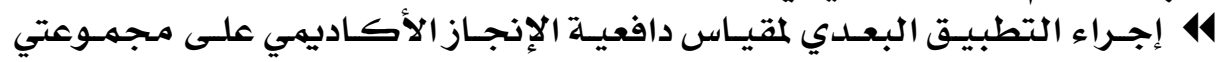

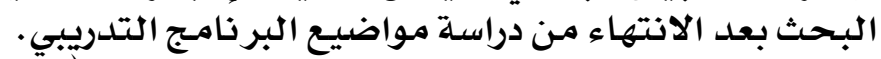

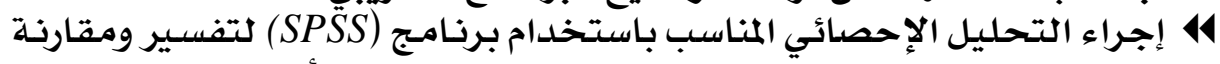

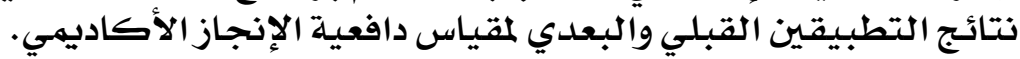

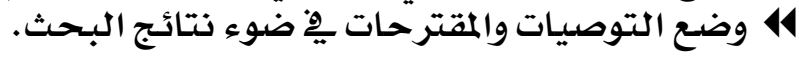

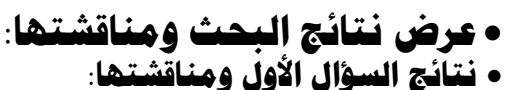

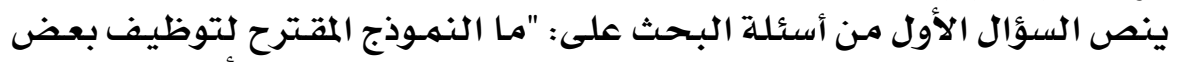

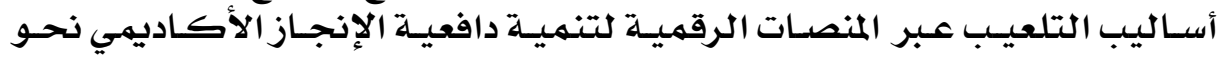

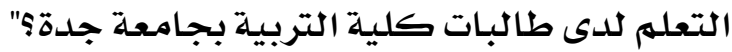

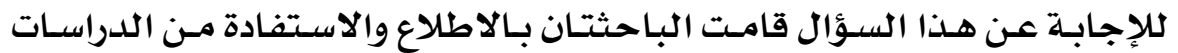

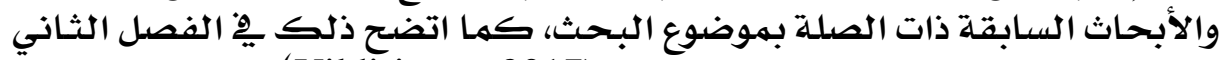

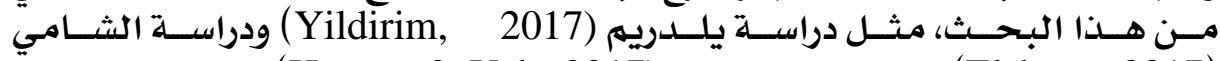

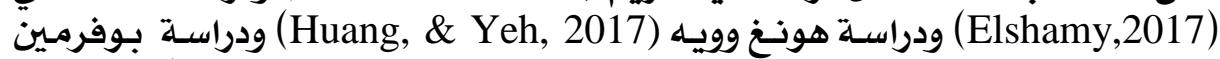

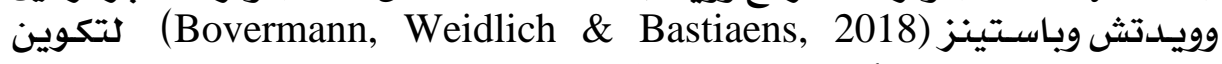

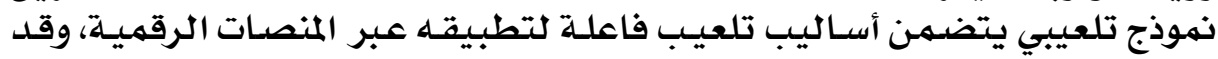

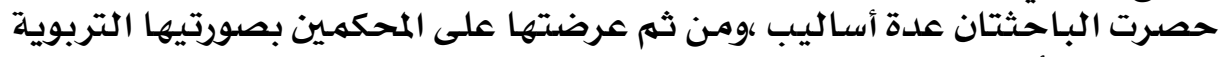

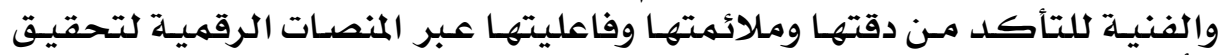

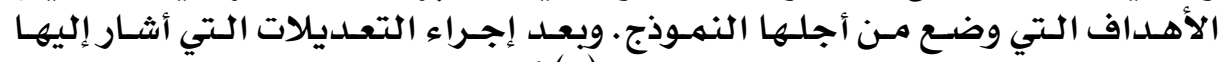

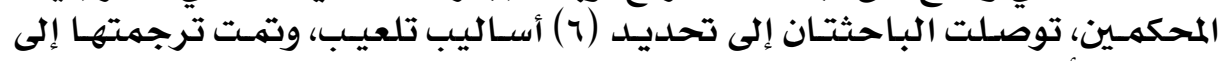

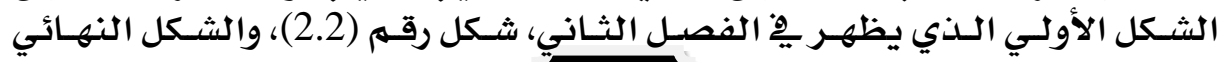




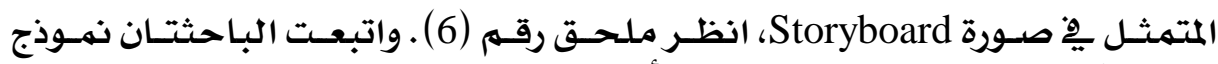

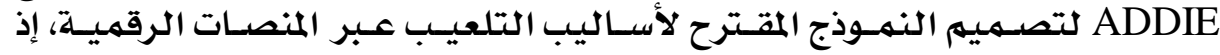

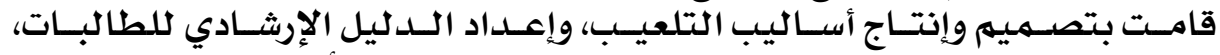

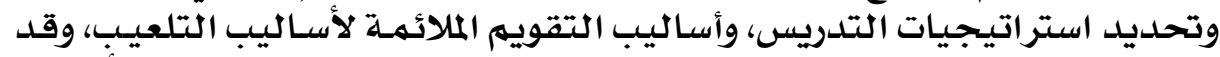

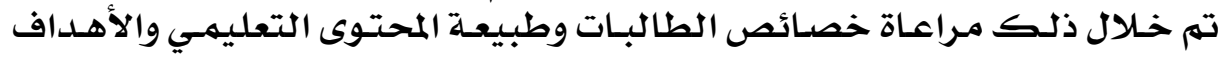

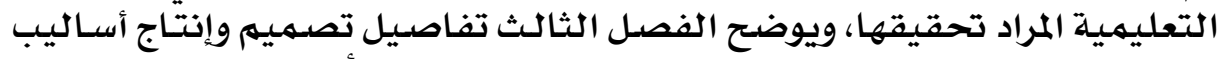

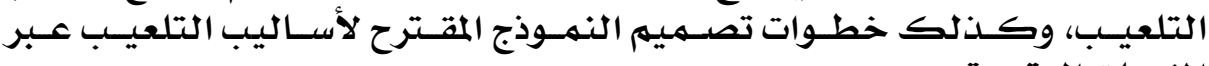

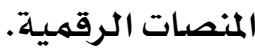

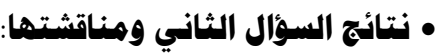

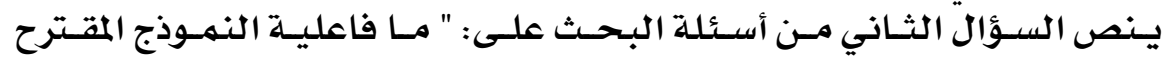

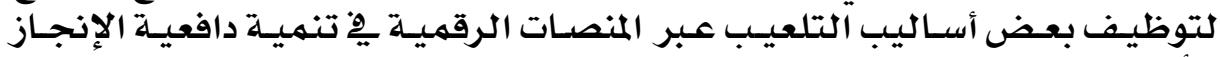

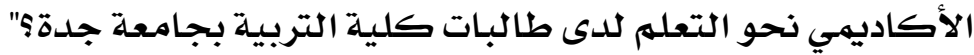

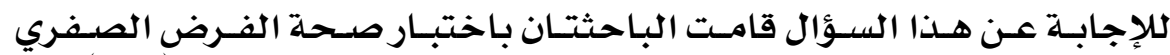

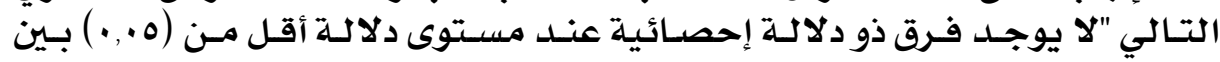

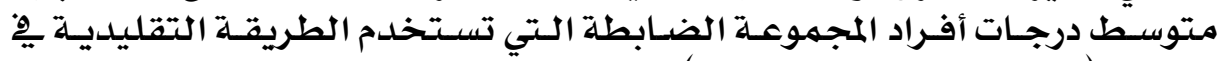

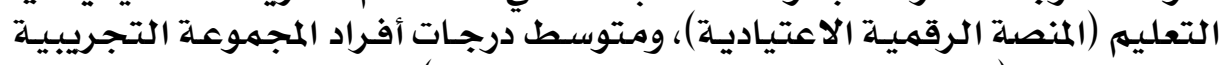

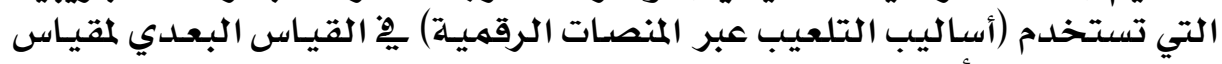

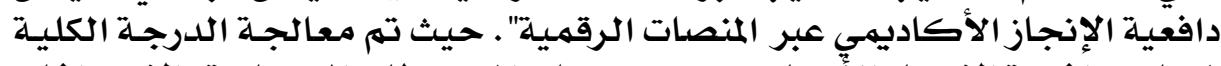

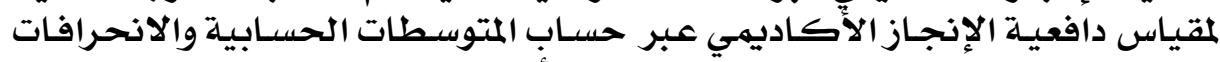

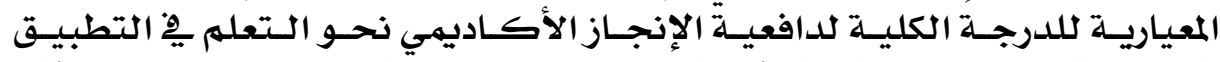

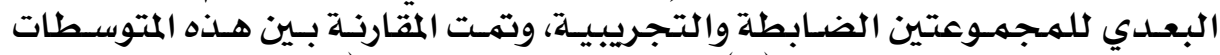

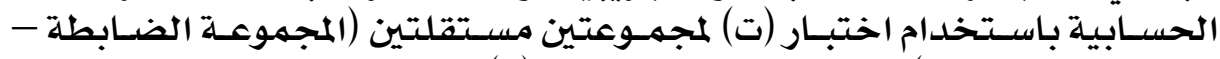

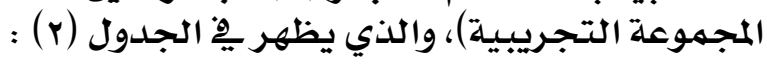

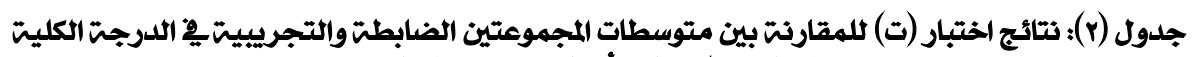
لدافعيتّا الإنجاز الأكاديمي نحو التعلم الفيط

\begin{tabular}{|c|c|c|c|c|c|c|c|c|}
\hline الإحصائيت & |الحريتي & قيمتةت & الالحراف & المتوسطب & العدد & المجموعت & التطبيق & المقارنت \\
\hline \multirow[b]{2}{*}{0.01} & \multirow{2}{*}{90} & \multirow{2}{*}{44.55} & 0.34 & 2.27 & \&7 & الضابطتة & البعدي & \multirow{2}{*}{ الألافعيت الإنجازيت الكليت التحو } \\
\hline & & & 0.09 & 4.58 & $\varepsilon 7$ & التجريبيت & البعدي & \\
\hline
\end{tabular}

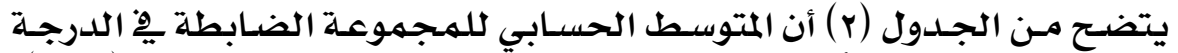

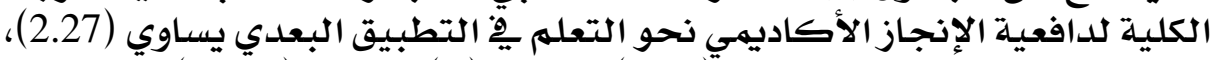

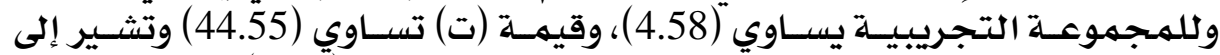

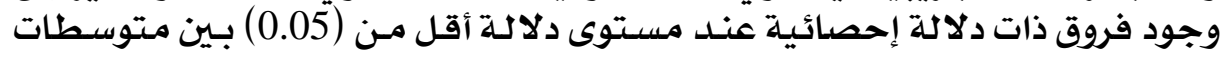

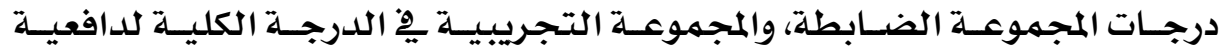

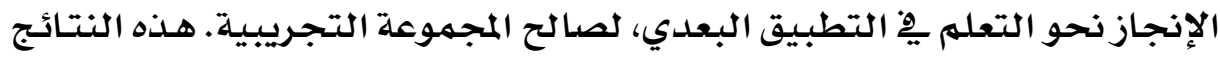

\section{$\varepsilon Y$}




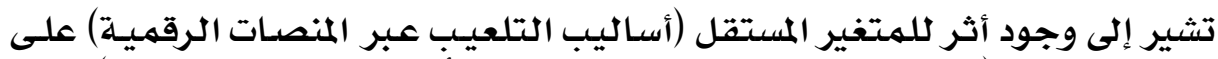

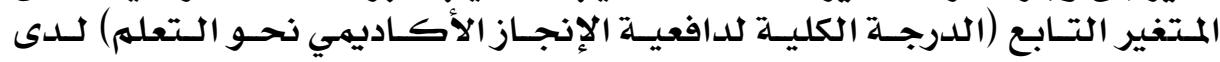
المجموعة التجريبية.

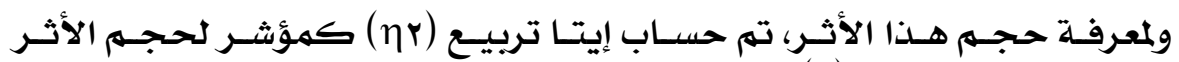

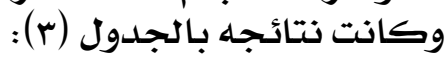

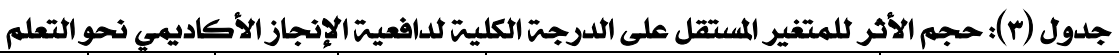

\begin{tabular}{|c|c|c|c|c|c|}
\hline حجم الأثر & ايتا & درجات & قيمتةت & المتغير التابع & المتغير المستقل \\
\hline كبير & -97 & 90 & 44.55 & الدارجت الكليتّ لدافعيت الإنجاز & أساليب التلعيب عبر المنصات \\
\hline
\end{tabular}

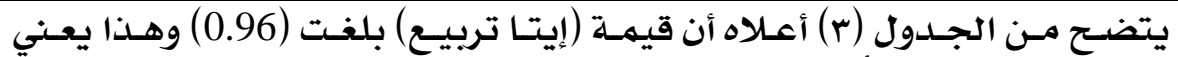

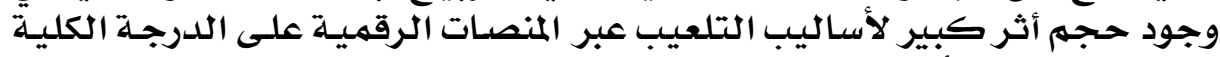

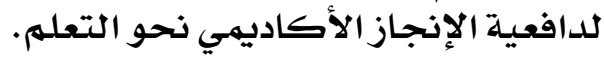

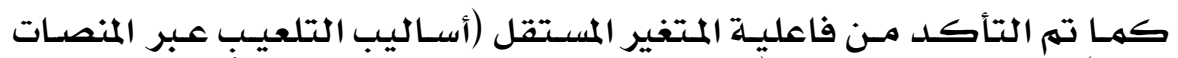

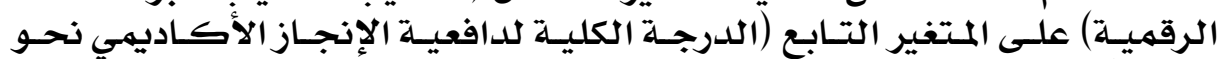

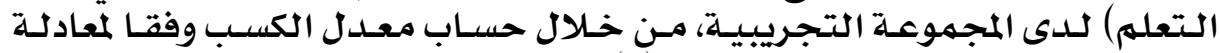

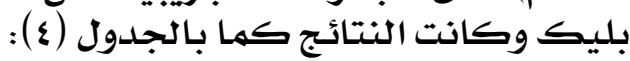

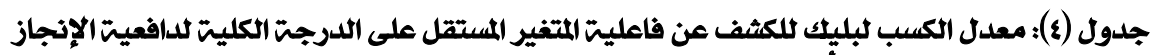

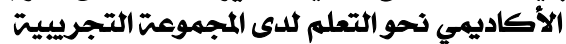

\begin{tabular}{|c|c|c|c|c|c|c|}
\hline \multirow{2}{*}{ الفاعليت } & \multirow{2}{*}{ معل الكسب } & \multicolumn{2}{|c|}{ المتوسط الحسابي } & \multirow{2}{*}{ العظمى الدرج } & \multirow{2}{*}{ المتغير التابع } & \multirow{2}{*}{ المتغير المستقل } \\
\hline & & بعدي & قبلي & & & \\
\hline كبيرة & 1.36 & 4.58 & 2.08 & 5 & الالإنجاز الكلية لداديمعية & أساليب التلعيب عبّر \\
\hline
\end{tabular}

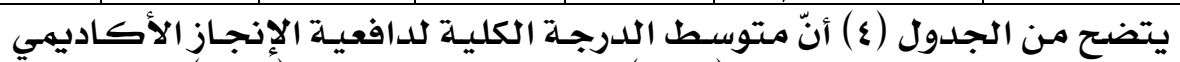

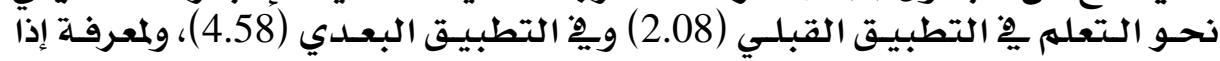

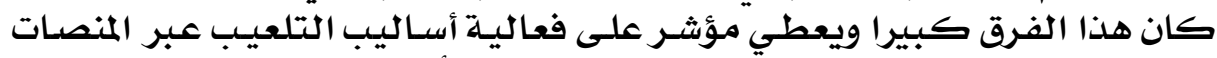

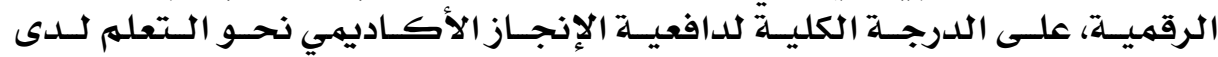

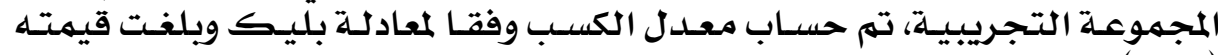

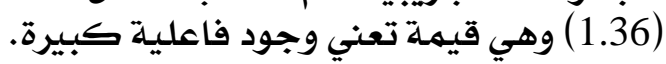

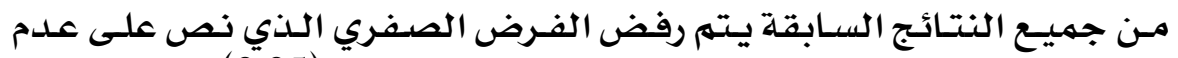

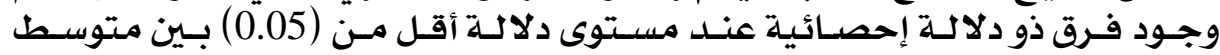

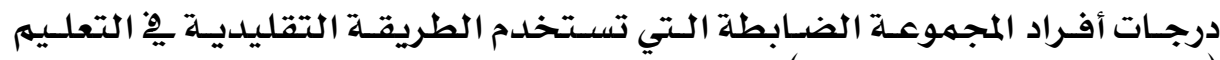

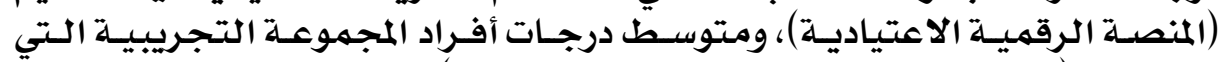

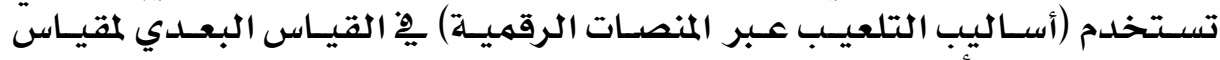

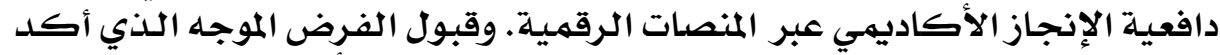

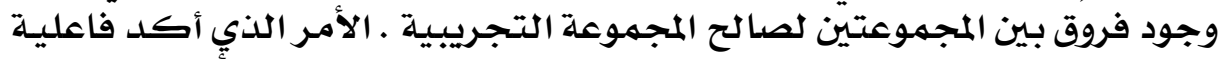

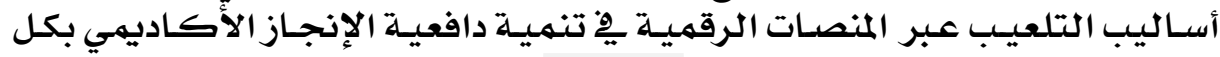

\section{$\varepsilon r$}




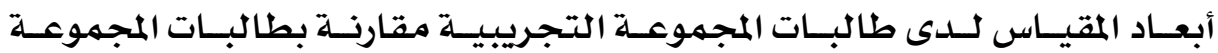

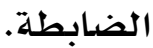

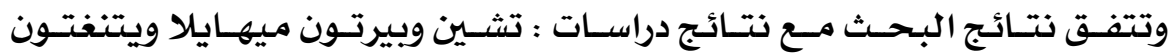
(Chen, Burton, Mihaela, \& Whittinghill 2015) وكارا وقوكتس (Turan, Avinc, Kara, \& Goktas,2016) و وراسـة هوانغ ويـه واسيردا وراسـة الشـامي (Huang, \& Yeh,2017)

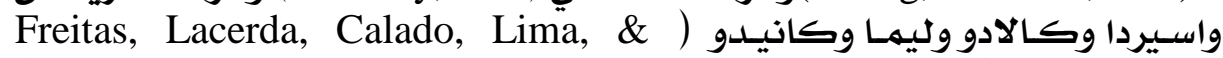
و و (Canedo, 2017

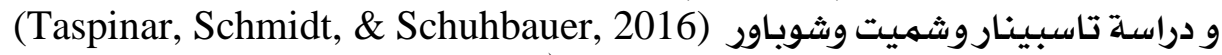

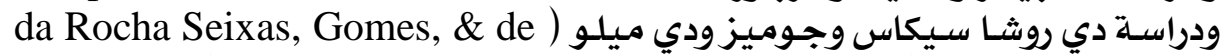
Ibáñez, Di-) دراسـة ايبـانيزوديز سيلويريو (Melo Filho, 2016 (Serio, \& Delgado-Kloos， 2014

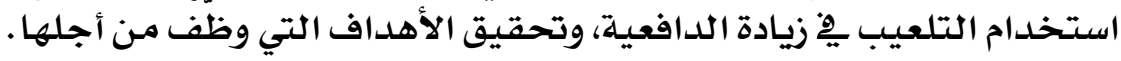

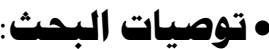

مِفوض ضوء نتائج البحثث أوصت البـاحثتان بالتوصيات الأتية:

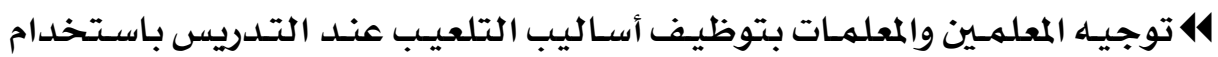

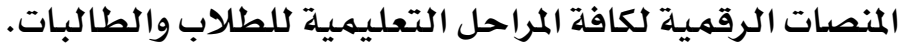

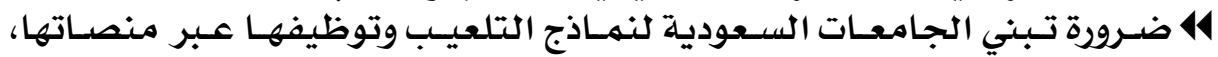

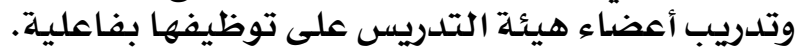

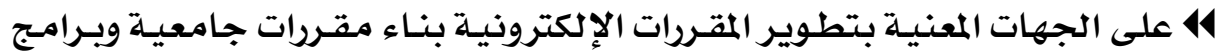

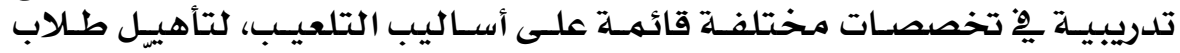

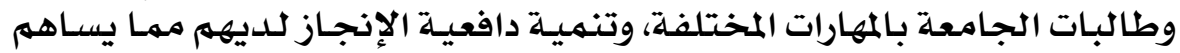

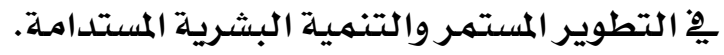

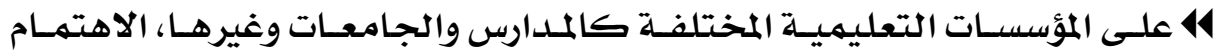

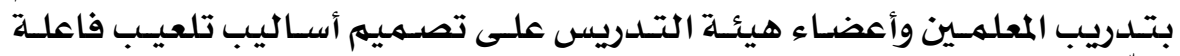

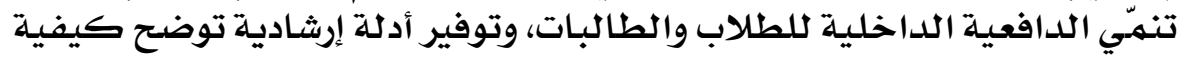

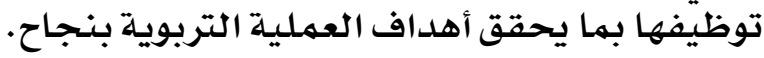

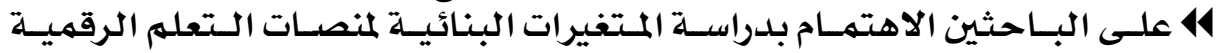

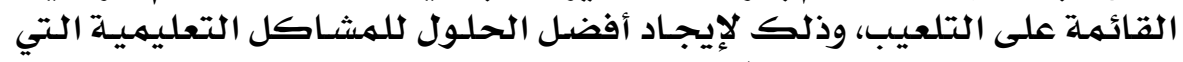

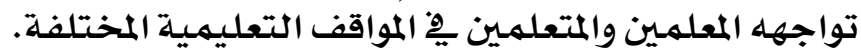

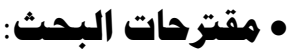

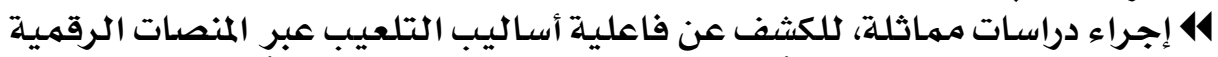

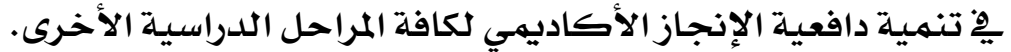

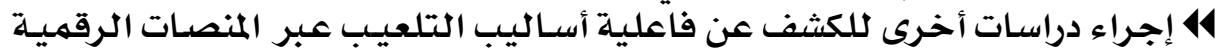

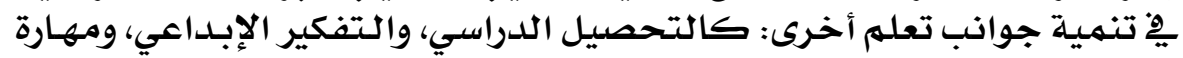
حل المشكلات ومهارات ما وراء تعبد المعرفة:

\section{$\varepsilon \varepsilon$}




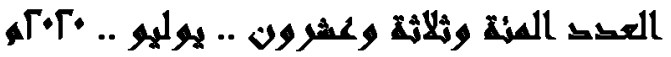

\ا إجـراء دراسـات مهـاثلـة تجريبيـة علـى جـامعـات أهليـة، ومنصــات رقميـة أخـرى

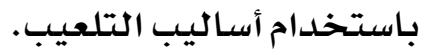

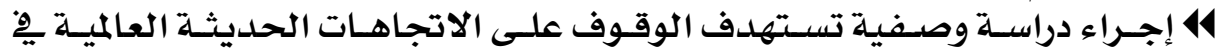

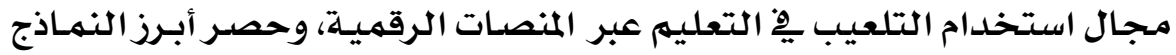

اناجحة.

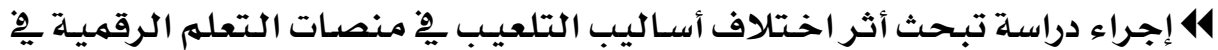

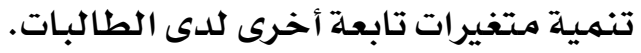

\section{• الرواجه: \\ • أولاً: المراجع العربية:}

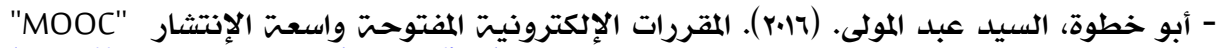

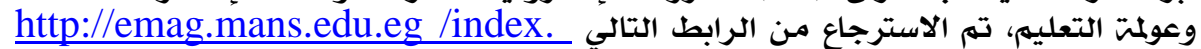
php?page=news\&task=show\&id=466

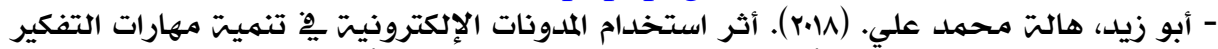

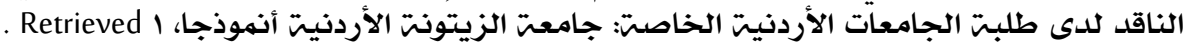
from_http://search.ebscohost.com.sdl.idm.oclc.org/login.aspx?direct=

true \&db=edsalm\&AN=edsalm.900720\&site=eds-live

- ابن الهدلق، عبدالله عبد العزيز.(19.r). التعليه بالترفيه: تصور مقترح لاستخدام التلعيب - Gamification

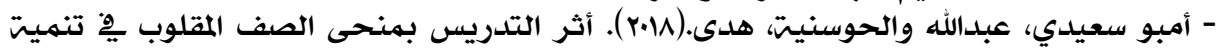

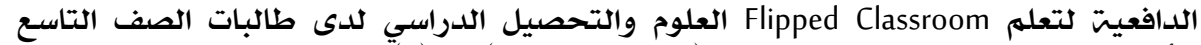

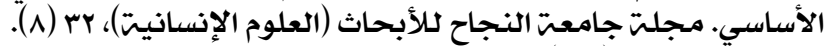

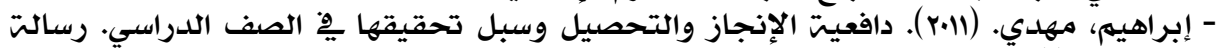

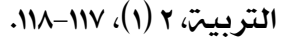

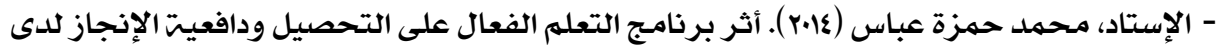

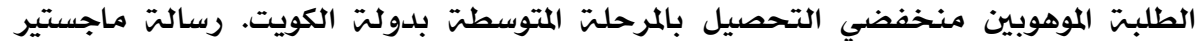

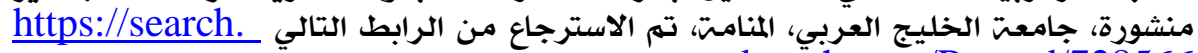

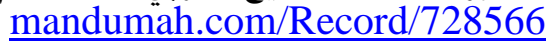

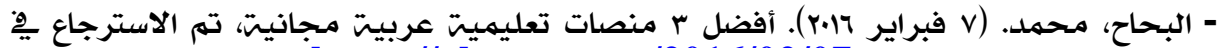

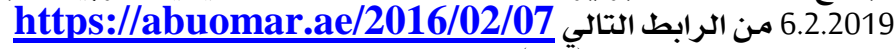

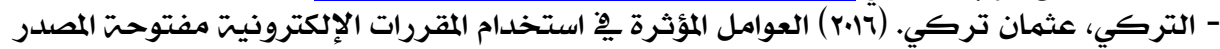

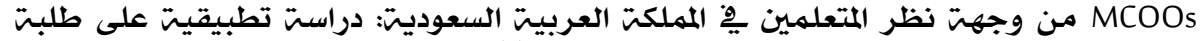

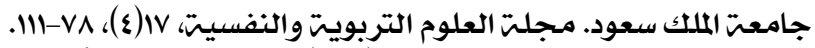

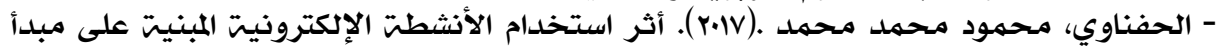

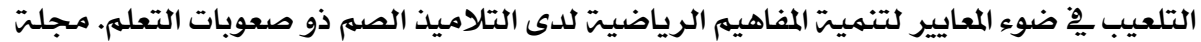

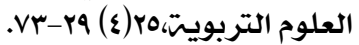

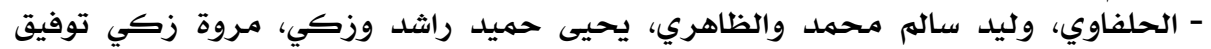

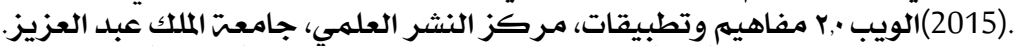

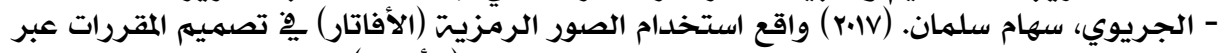

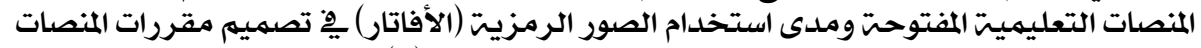

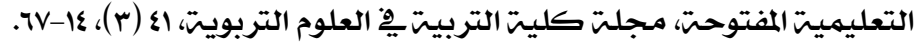

\section{$\varepsilon \odot$}




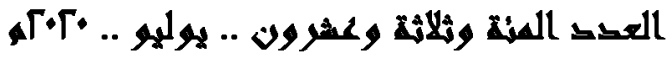

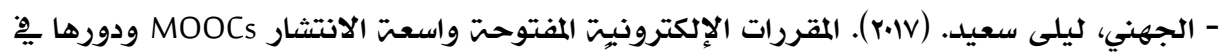

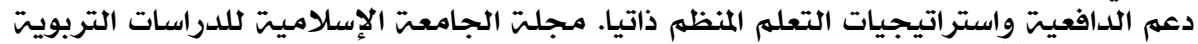

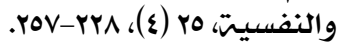

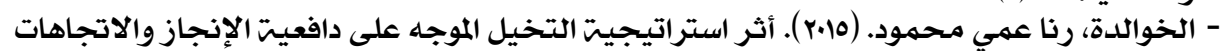

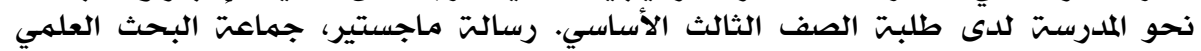

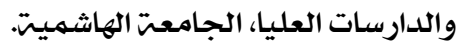

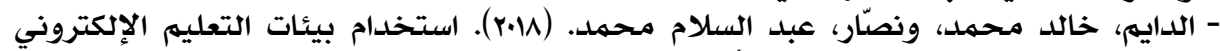

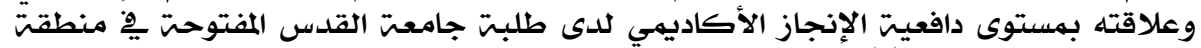

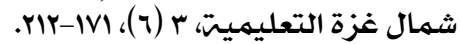

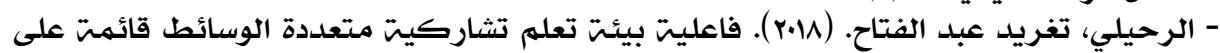

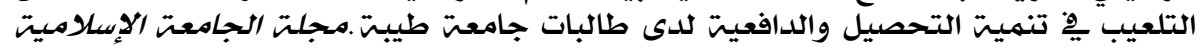

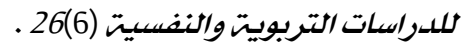

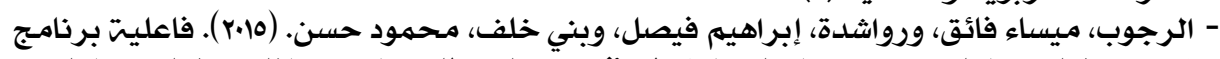

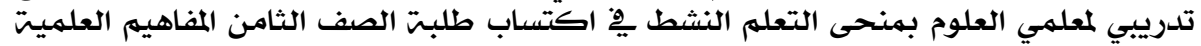

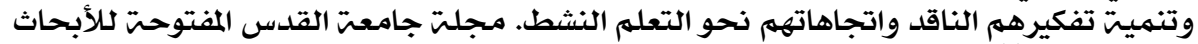

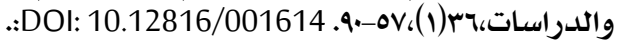

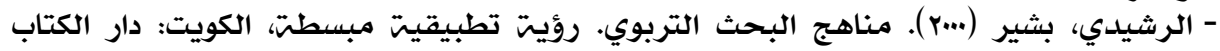

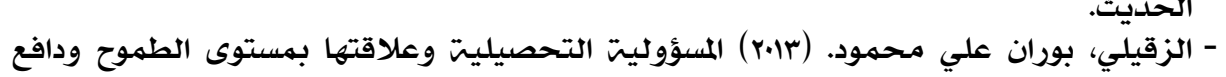

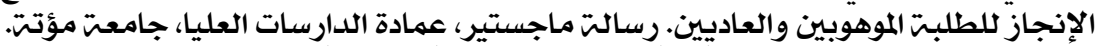

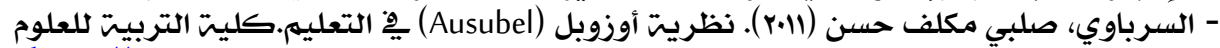

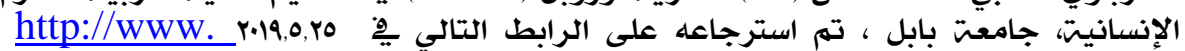
uobabylon.edu.iq/uobColeges/lecture.aspx? fid=10\&depid=6\&lcid=16

750

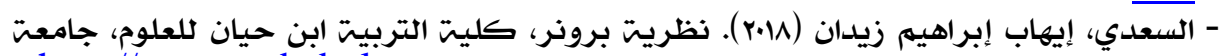

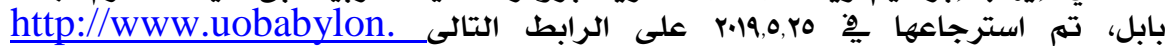
edu.iq/uobcoleges/lecture.aspx?fïd=21\&lcid=80353

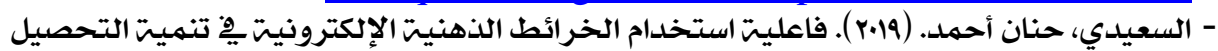

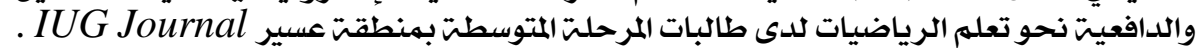
of Educational \& Psychological Studies, 27(1), 300-324. Retrieved from http://search.ebscohost.com.sdl.idm.oclc.org/login.aspx?direct= true\&db=awr\&AN=134474964\&site=eds-live

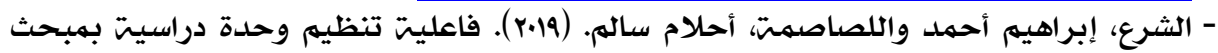

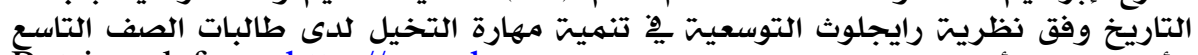

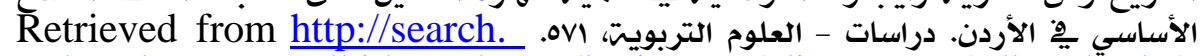
ebscohost.com.sdl.idm.oclc.org/login.aspx?direct=true \&db=ës AN=edseds.949015\&site=eds-live

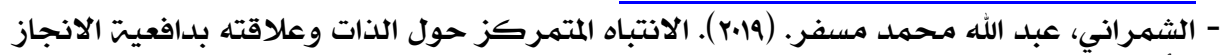

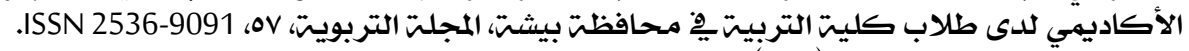

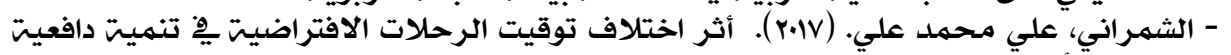

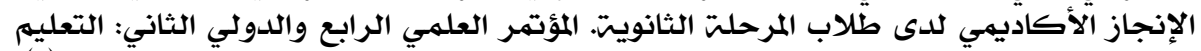

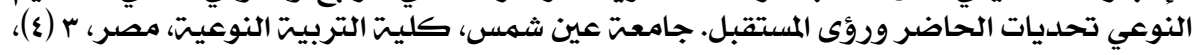

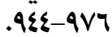

\section{7}




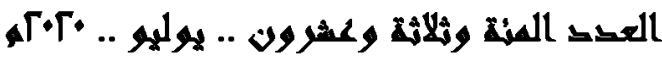

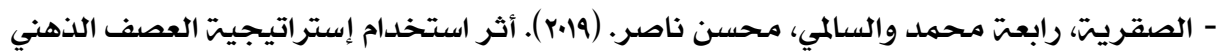

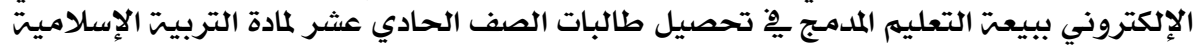

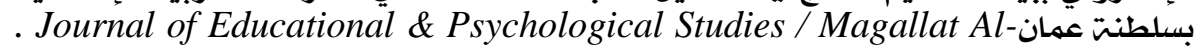
Dirasat Al-Tarbawiyyat Wa-Al-Bafsiyyat, 13(3), 516-537. https://doiorg.sdl.idm.oclc.org/10.24200/jeps.vol13iss3pp516-537

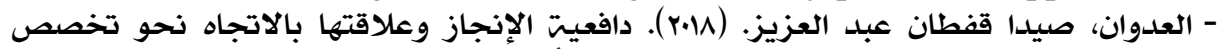

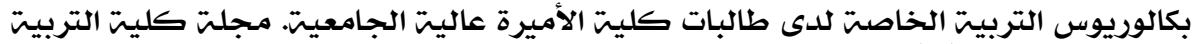

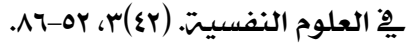

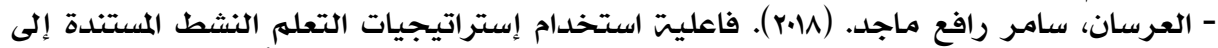

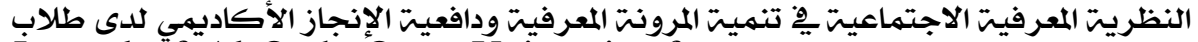

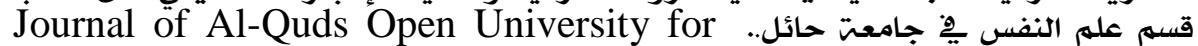
Educational \& Psychological Research \& Studies, 5(18), 159-177. Retrieved from http://search.ebscohost.com.sdl.idm.oclc.org/login. aspx? direct=true \&db=awr\&AN=127953964\&site=eds-live

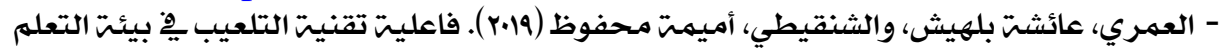

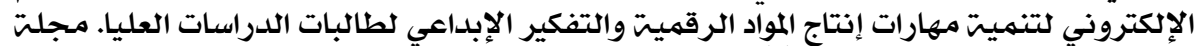

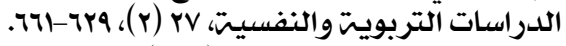

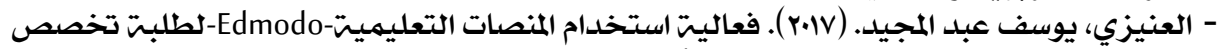

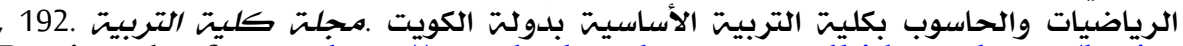
Retrieved from http://search.ebscohost.com.sdl.idm.oclc.org/login. aspx? direct=true\&db=edseds\&AN=edseds.844035\&site=eds-live

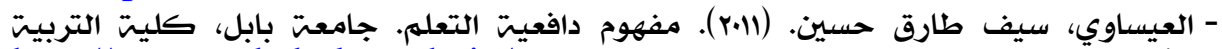

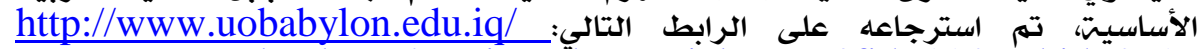
uobcoleges/service_showarticle.aspx?fid=11\&pubid=2716

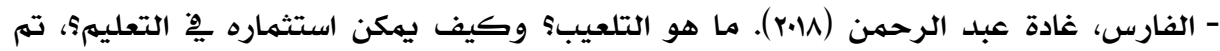

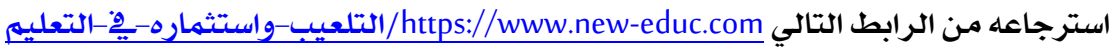

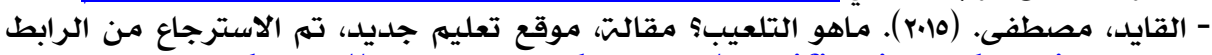
https://www.new-educ.com/gamification-education التالي ماهو

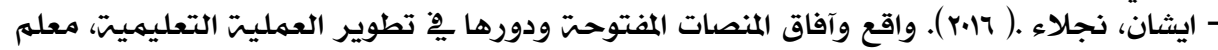

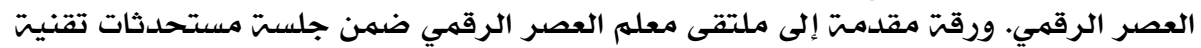

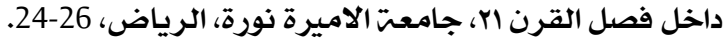

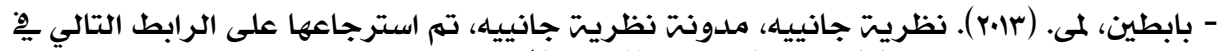
/ http://gagnetheory.blogspot.com r.19,0,ro - بدوي، حنان وعبد الجليل، بدر. (rا.r). العوامل المؤثرة على دافعيت الإنجاز للتحصيل الدراسي.

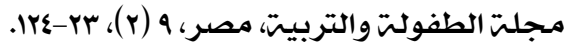

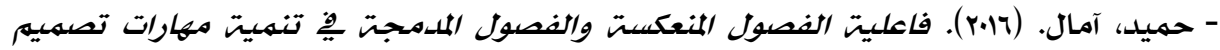

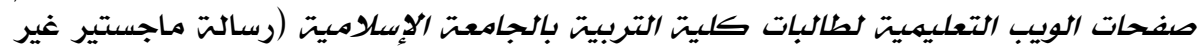

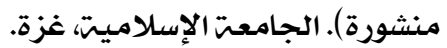

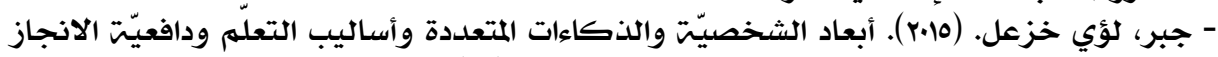

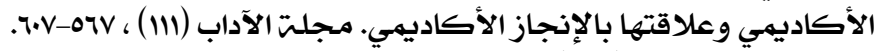

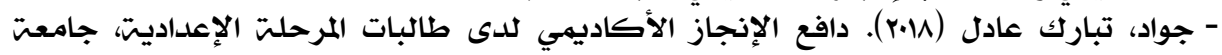
القادسيـت. 


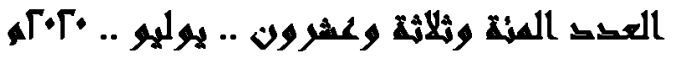

- خلف الله، محمد جابر ( سا.r)، التعليه بشبكات التواصل الاجتماعي، تم استرجاعه هِّ

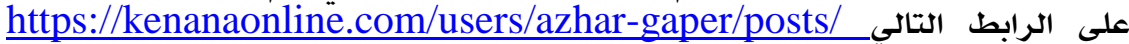

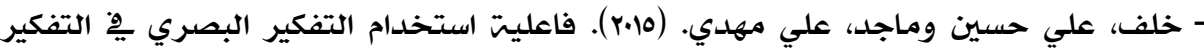

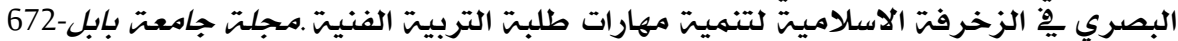
689,(2) 23.

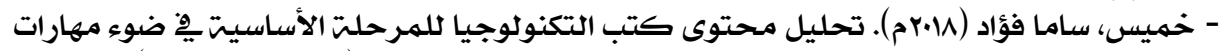

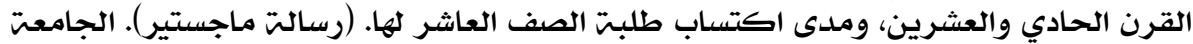

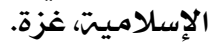

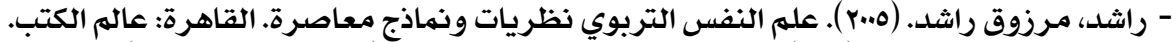

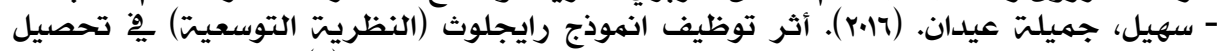

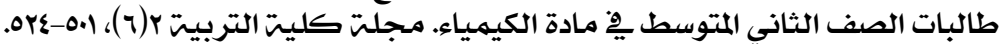

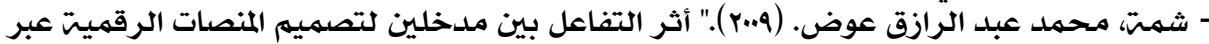

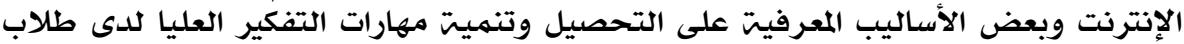

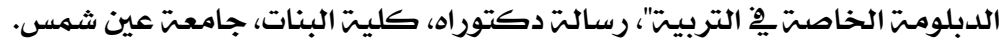

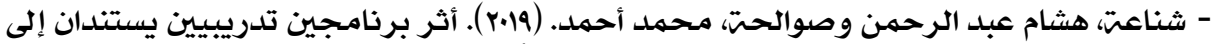

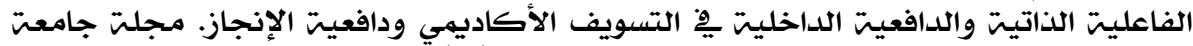

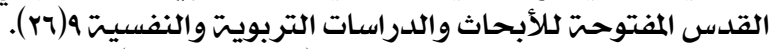

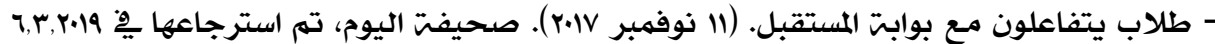
ملى الرابط التالي

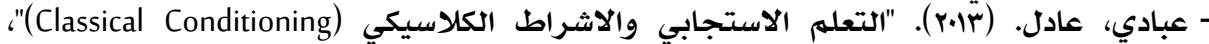

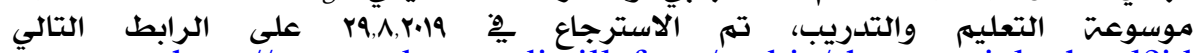
http://www.edutrapedia.illaf.net/arabic/show_article.thtml?id

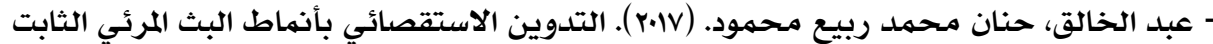

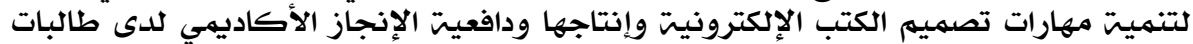

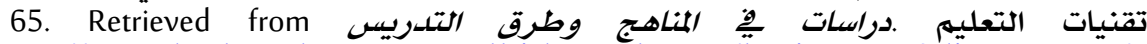
http://search.ebscohost.com.sdl.idm.oclc.org/login.aspx?direct=true\& $\mathrm{db}=$ edseds\&AN=edseds.833848\&site=eds-live

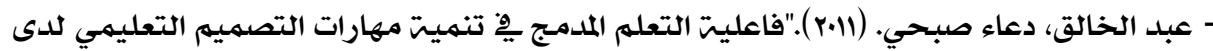

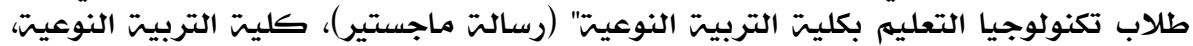

جامعت بنها.

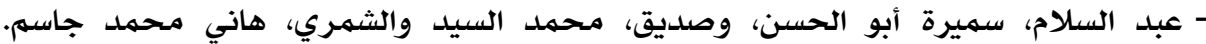

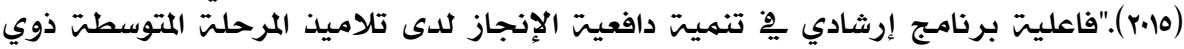

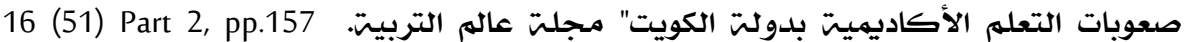

Retrieved from https://platform.almanhal.com/Files/2/95007

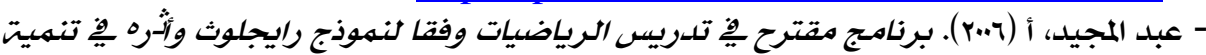

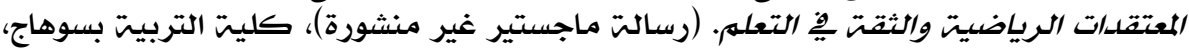

- عزمي، نبيل جاد. (عا.r). بيئات التعلهم التفاعليت. طا. القاهرة: دار الفكر العربي.

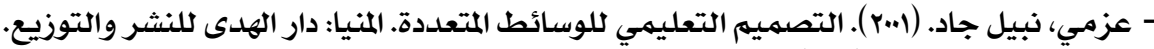

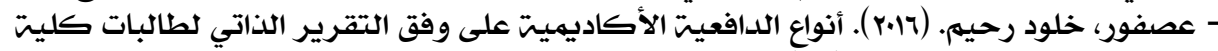

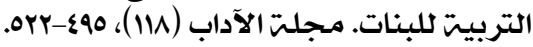

\section{$\varepsilon \wedge$}




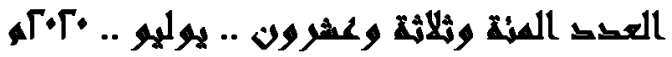

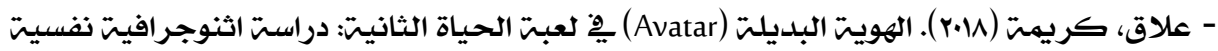

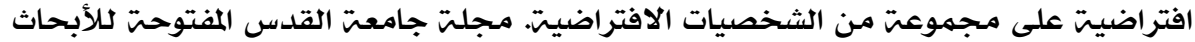

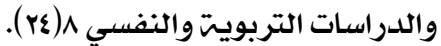

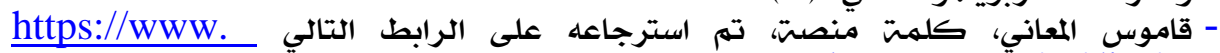
almaany.com/ar/dict/ar

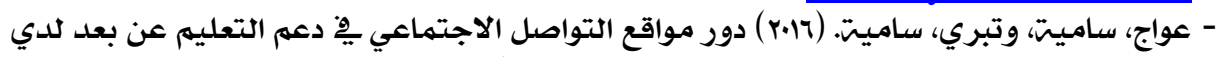

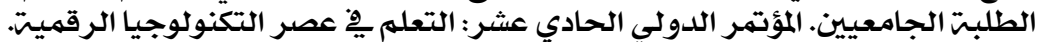

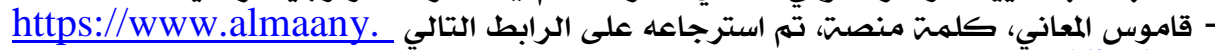
com/ar/dict/ar-

https://WwW. قاموس المعاني، كلمت دافع الإنجاز، تم استرجاعه على الرابط التالي almaany.com/ar/dict/ar-

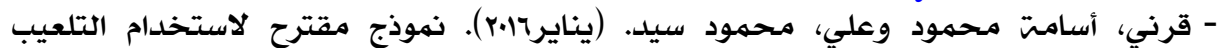
بالجامعات المصريت. ورقت عمل مقدمدي، (gamification)

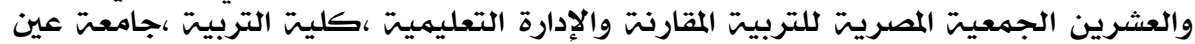

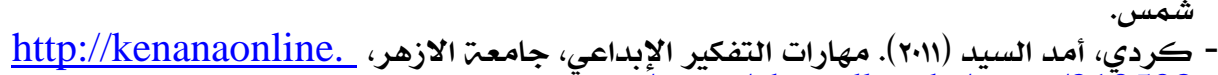
com/users/ahmedkordy/posts/210508

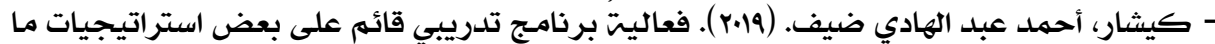

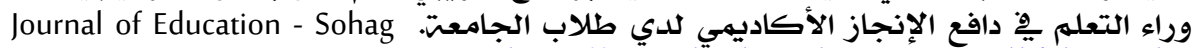
University, 58, 133. Retrieved from http://search.ebscohost.com.sdl.idm.oclc. org/login.aspx?direct=true\&db=awr\&AN=134861604\&site=eds-live

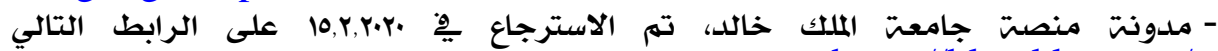
https://blog.kkux.org/

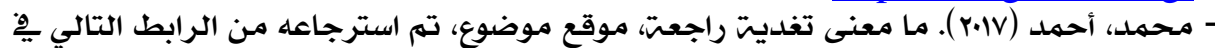
https://mawdoo3.com/r. , , , r.19

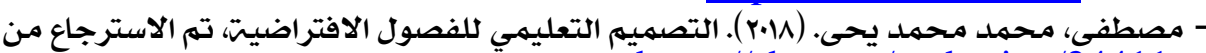
https://shms.sa/authoring/24411- مطاوع، ضياء الدين محمد والخليفتة، حسن جعفر (عا.r). مبادئ البحث يِّ العلوم التربويت

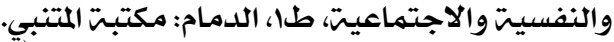

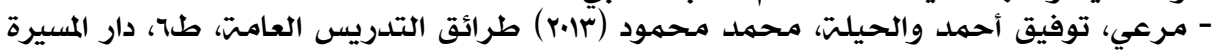
للنشر، عمان. توفيق

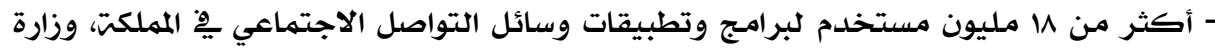

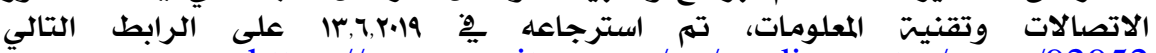
https://WwW.mcit.gov.sa/en/media-center/news/92952

• ثانياً - المراجع الأجنبية:

- Abdulrahman, A. S., \& Hui, X. Implication of Motivation Theories on Teachers Performance in the Context of Education System in Tanzania.

- Alario-Hoyos, C., Estévez-Ayres, I., Pérez-Sanagustín, M., Kloos, C. D., \& Fernández-Panadero, C. (2017). Understanding learners' motivation and learning strategies in MOOCs. The International Review of Research in Open and Distributed Learning, 18.( $\left.{ }^{\top}\right)$

\section{$\varepsilon 9$}




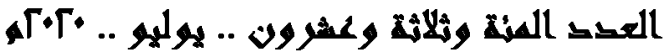

- Alabbasi, D. (2017). Exploring Graduate Students' Perspectives towards Using Gamification Techniques in Online Learning. Turkish Online Journal of Distance Education, 18(3), n3.

- Aladağ, E. (2010). The effects of GIS on students' academic achievement and motivation in seventh-grade social studies lessons in Turkey. International Research in Geographical and Environmental Education, 19(1), 11-23.

- Agrawal, M., \& Teotia, A. K. (2015). Academic achievement and selfconcept of secondary level students. International Education and Research Journal, 1(3), 26-33.

- Aktas, E., \& Yurt, S. U. (2017). Effects of Digital Story on Academic Achievement, Learning Motivation and Retention among University Students. International Journal of Higher Education, 6(1), 180-196. Retrieved from http://search.ebscohost.com.sdl.idm.oclc.org/login. aspx?direct $=$ true $\& d \mathrm{~b}=$ eric $\& A N=E J 1126025 \&$ site $=$ eds-live

- Aldemir, T., Celik, B., \& Kaplan, G. (2018). A qualitative investigation of student perceptions of game elements in a gamified course. Computers in Human Behavior, 78, 235-254. https://doiorg.sdl.idm.oclc.org/10.1016/j.chb.2017.10.001

- Alexe, I., Zaharescu, L., \& Apostol, S. (2013). Gamification of Learning and Educational Games. Proceedings of the 9th International Scientific Conference eLearning and Software for Education (pp. 6772). Bucharest, Romania: Editura Universitatii Nationale de Aparare.

- Al-Samarraie mH, Teng Bee, K, Alzahrani, A \& Alalwan, N (2017): E-learning continuance satisfaction in higher education: a unified perspective from instructors and students, Studies in Higher Education, DOI: 10.1080/03075079.2017.1298088

- AlWashmi, R., Baines, M., Organ, S., Hopkins., and Balanchedfield, P. (2014). Mathematics Problem Solving Through Collaboration: Game design and Adventure. In C. Buch (eds.) Proceedings of the 8th European Conference on Games based Learning. (pp.1-9).UK: Academic Conferences and Publishing International limited Reading.

- Annansingh, F. (2018). An Investigation Into the Gamification of ELearning in Higher Education. In Gamification in Education: Breakthroughs in Research and Practice (pp. 174-190). IGI Global.

- Areepattamannil, S. (2011). Academic self-concept, academic motivation, academic engagement, and academic achievement: A mixed methods study of Indian adolescents in Canada and India (Doctoral dissertation).

- Arhipova ,Alina. Gamification in UX. Missions and Challenges, Tubik blog, retrieved 27.5.2019 from https://tubikstudio.com/gamificationin-ux-missions-and-challenges/ 
- Arnavut, A., Bicen, H., \& Nuri, C. (2019). Students' Approaches to Massive Open Online Courses: The Case of Khan Academy. BRAIN. Broad Research in Artificial Intelligence and Neuroscience, 10(1), 8290.

- Azevedo, J., Padrão, P., Gregório, M. J., Almeida, C., Moutinho, N., Lien, N., \& Barros, R. (2019). A Web-Based Gamification Program to Improve Nutrition Literacy in Families of 3-to 5-Year-Old Children: The Nutriscience Project. Journal of nutrition education and behavior, 51(3), 326-334.

- Bandura, A. (1993). Perceived Self-Efficacy in Cognitive Development and Functioning. Educational psychologist, 28 (2), 117148.

- Bunchball, Inc (2010). Gamification 101: An Introduction to the Use of Game Dynamics to Influence Behavior (White Paper).

- Burke, M. and Hiltbrand, T. (2011). How Gamification Will Change Business Intelligence. Business Intelligence Journal, 16 (2), 8-16.

- Burke, B. (2012). Gamification trends and strategies to help prepare for the future. Gartner, Paris.

- Bovermann, K., Weidlich, J., \& Bastiaens, T. (2018). Online learning readiness and attitudes towards gaming in gamified online learning-a mixed methods case study. International Journal of Educational Technology in Higher Education, 15(1), 27.

- Chen, B., \& Bryer, T. (2012). Investigating instructional strategies for using social media in formal and informal learning. The International Review of Research in Open and Distributed Learning, 13(1), 87-104.

- C. Lampe, Gamification and Social Media, in the gameful world: approaches, issues, applications, S. Deterding and S.P. Walz, Editors. 2014, MIT Press: Cambridge, Massachusetts.

- Caton, H., \& Greenhill, D. (2013). The Effects of Gamification on Student Attendance and Team Performance in a Third-Year Undergraduate Game Production Module. Proceedings of the European Conference on Games Based Learning (pp. 88-96). Porto, Portugal: Academic Conferences and Publishing International Limited.

- Chauhan, J., Taneja, S., and Goel, A. (2015). Enhancing MOOC with augmented reality, adaptive learning and gamification. In MOOCs, Innovation and Technology in Education (MITE). (pp. 348-353).

- Chen, Y., Burton, T., Mihaela, V., \& Whittinghill, D. (2015). Cogent: a case study of meaningful gamification in education with virtual currency. iJET-International Journal of Emerging Technologies in Learning, 10, 133-147.

- Cheong, C., Cheong, F., \& Filippou, J. (2013, June). Quick Quiz: A Gamified Approach for Enhancing Learning. In PACIS (p. 206) 
- Christensen, G., Steinmetz, A., Alcorn, B., Bennett, A., Woods, D., \& Emanuel, E. J. (2014). The MOOC phenomenon: Who takes massive open online courses and why? 2013. Retrieved October, 12.

- Conole, G. G. (2015). MOOCs as disruptive technologies: strategies for enhancing the learner experience and quality of MOOCs. Revista de Educación a Distancia, (39).

- da Rocha Seixas, L., Gomes, A. S., \& de Melo Filho, I. J. (2016). Effectiveness of gamification in the engagement of students. Computers in Human Behavior, 58, 48-63.

- Denden, M., Tlili, A., Essalmi, F., \& Jemni, M. (2017, October). Educational gamification based on personality. In 2017 IEEE/ACS 14th International Conference on Computer Systems and Applications (AICCSA) (pp. 1399-1405). IEEE.

- De-Marcos, L., García-López, E., García-Cabot, A., Medina-Merodio, J. A., Domínguez, A., Martínez-Herráiz, J. J., \& Diez-Folledo, T. (2016). Social network analysis of a gamified e-learning course: Small-world phenomenon and network metrics as predictors of academic performance. Computers in Human Behavior, 60, 312-321.

- De-Marcos, L., Garcia-Cabot, A., \& Garcia-Lopez, E. (2017). Towards the social gamification of e-Learning: A practical experiment. International journal of engineering education, 33(1), 6673.

- Deterding, S., Khaled, R., Nacke, L., \& Dixon, D. (2011). Gamification: Toward a Definition. CHI 2011, Gamification Workshop Proceedings.

- Dicheva, D., Dichev, C., Agre, G., \& Angelova, G. (2015). Gamification in education: A systematic mapping study. Educational Technology \& Society, 18(3), 75-88.

- Domínguez, A., Saenz-de-Navarrete, J., de-Marcos, L., FernándezSanz, L., Pagés, C. and Martínez-Herráiz, J.-J. (2013). Gamifying Learning Experiences: Practical Implications and Outcomes. Computers \& Education, 63, 380-392.

- Duggal, K., Srivastav, A., \& Kaur, S (2014). Gamified Approach to Database Normalization. International Journal of Computer Applications, 93(4), 47- 53.

- El Tantawi, M., Sadaf, S., \& AlHumaid, J. (2018). Using gamification to develop academic writing skills in dental undergraduate students. European Journal of Dental Education, 22(1), 15-22.

- Elshemy, N. (2017, May). Impact of Gamification strategy on academic achievement and achievement motivation toward learning. In Proceedings of Teaching and Education Conferences (No. 4907055). International Institute of Social and Economic Sciences. 


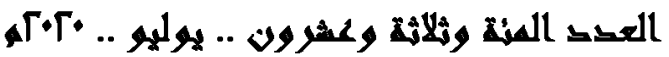

- Etuk, N. M., \& Clegg, R. L. (2007). U.S. Patent No. 7,257,367. Washington, DC: U.S. Patent and Trademark Office.

- Evans, P. (2015). Self-determination theory: An approach to motivation in music education. Musicae Scientiae, 19(1), 65-83.

- Freitas, S. A. A., Lacerda, A. R., Calado, P. M., Lima, T. S., \& Canedo, E. D. (2017, October). Gamification in education: A methodology to identify student's profile. In 2017 IEEE Frontiers in Education Conference (FIE) (pp. 1-8). IEEE.

- Galbis-Córdova, A., Martí-Parreño, J., \& Currás-Pérez, R. (2017). Higher Education Students' Attitude Towards the Use of Gamification for Competencies Development. Journal of E-Learning \& Knowledge Society, 13(1), 129-146. Retrieved from http://search.ebscohost.com. sdl.idm.oclc.org/login.aspx?direct $=$ true $\& d \mathrm{db}=\mathrm{ehh} \& \mathrm{AN}=121262055 \& \mathrm{si}$ te=eds-live

- Gamified learning in higher education: A systematic review of the literature. Computers in Human Behavior, 87, 192-206.

- Gardner, S. J. (2003) Factors influencing achievement motivation in highly motivated, dedicated and enthusiastic high school learners. Unpublished doctoral dissertation, Union Institute and University.

- Garud, R. and Kumaraswamy, A. (2005). Vicious and Virtous Circles in the Management of Knowledge: The Case of Infosys Technologies. MIS Quarterly, 29 (1), 9-33.

- González, C., \& Carreño, A. (2014). Methodological Proposal for Gamification in the Computer Engineering Teaching. Proceedings of the International Symposium on Computers in Education SIIE (pp. 2934). Logroño, Spain: IEEE

- Granter, C.P., (2012, November 27) Gamification Trends and Strategies to Help Prepare for the Future" on November 28

- Herzig, P. (2014). Gamification as a Service: Conceptualization of a Generic Enterprise Gamification Platform. Dresden, Technische Universität Dresden (Doctoral dissertation, Ph. D. Dissertation).

- Hakak, S., Noor, N. F. M., Ayub, M. N., Affal, H., Hussin, N., ahmed, E., \& Imran, M. (2019). Cloud-assisted gamification for education and learning - Recent advances and challenges. Computers and Electrical Engineering, 74, 22-34. https://doi-org.sdl.idm.oclc.org/10.1016/j. compeleceng.2019.01.002

- Huang, W. H., \& Soman, D. (2013). A Practitioner's Guide to Gamification of Education. Research Reports Series, Behavioural Economics in Action. Rotman School of Management, University of Toronto. December 10.

- Huang, L. Y., \& Yeh, Y. C. (2017). Meaningful gamification for journalism students to enhance their critical thinking 


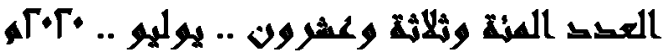

skills. International Journal of Game-Based Learning (IJGBL), 7(2), 47-62.IGI Global.

- Ibanez, M. B., Di-Serio, A., \& Delgado-Kloos, C. (2014). Gamification for engaging computer science students in learning activities: A case study. IEEE Transactions on learning technologies, 7(3), 291-301.

- J. Lee and C.J. Bonk, Social network analysis of peer relationships and online interactions in a blended class using blogs. The Internet and Higher Education, 2016. 28: pp. 35-44.

- Jaipal-Jamani, K., \& Figg, C. (2018). Application of gamification to blended learning in higher education. In Encyclopedia of Information Science and Technology, Fourth Edition (pp. 3238-3247.

- Jakubowski, M. (2014, March). Gamification in Business and Education "Project of Gamified Course for University Students. In Developments in Business Simulation and Experiential Learning: Proceedings of the Annual ABSEL conference (Vol. 41).

- Jordan, K. (2014). Initial trends in enrolment and completion of massive open online courses. The International Review of Research in Open and Distributed Learning, 15.( ( )

- Jung, Y., \& Lee, J. (2018). Learning engagement and persistence in massive open online courses (MOOCS). Computers \& Education, 122, 9-22.

- Kady, H. R., \& Vadeboncoeur, J. A., PhD. (2017). Massive Open Online Courses (MOOC). Salem Press Encyclopedia. Retrieved from http://search.ebscohost.com.sdl.idm.oclc.org/login.aspx?direct=true\&d $\mathrm{b}=\mathrm{ers} \& \mathrm{AN}=89550604 \&$ site $=$ eds-live

- Karl, M. Kaap. (2012). The Gamification of Learning and Instruction. Pfeiffer.ISBN-10:1118096345

- Khalaila, R. (2015). The relationship between academic self-concept, intrinsic motivation, test anxiety, and academic achievement among nursing students: Mediating and moderating effects. Nurse Education Today, 35(3), 432-438.

- Kim, K., \& Ahn, S. J. (2017). Rewards that undermine customer loyalty? A motivational approach to loyalty programs. Psychology \& Marketing, 34(9), DOI: 842-852. / •, l . r mar.21026

- Kim, B. (2015). Designing Gamification in the Right Way. Library Technology Reports, 51(2), 29-35.

- Kiryakova, G., Angelova, N., \& Yordanova, L. (2014). Gamification in education. Proceedings of 9th International Balkan Education and Science Conference.

- Krause, M., Mogalle, M., Pohl, H., \& Williams, J. J. (2015, March). A playful game changer: Fostering student retention in online education

\section{$0 \varepsilon$}




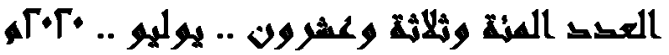

with social gamification. In Proceedings of the Second (2015) ACM Conference on Learning@ Scale (pp. 95-102). ACM.

- KISSAU, S., Chuang, W. A. N. G., RODGERS, M., HAUDECK, H., \& BIEBRICHER, C. (2019). The motivational profile of adolescent foreign language learners: An international comparison. International Journal of Language Studies, 13(1). pp. 19-40.

- Kusuma, G. P., Wigati, E. K., Utomo, Y., \& Suryapranata, L. K. P. (2018). Analysis of Gamification Models in Education Using MDA Framework. Procedia Computer Science, 135, 385-392.

- Lauren Phillips (2014) Module 7: Gamification design framework, Kevin Werbach, University of Pennsylvania Wharton School. P 12.

- Leal, C., Silva, R., \& Rodrigues, R. (2018, October). Gamification in Management Education: Systematic Literature Review and a Proposal of a Conceptual Research Model. In Proceedings of the European Conference on Games Based Learning (pp. 335-343).

- Legaki, N. Z., Xi, N., Hamari, J., \& Assimakopoulos, V. (2019, January). Gamification of The Future: An Experiment on Gamifying Education of Forecasting. In Proceedings of the 52nd Hawaii International Conference on System Sciences.

- Littlejohn, A., Hood, N., Milligan, C., \& Mustain, P. (2016). Learning in MOOCs: Motivations and self-regulated learning in MOOCs. The Internet and Higher Education, 29, 40-48.

- Mizuno, K., Tanaka, M., Ishii, A., Tanabe, H. C., Onoe, H., Sadato, N., and Watanabe, Y. (2008). The neural basis of academic achievement motivation. Neuroimage, 42(1), 369-378.

- Morales, M., Amado-Salvatierra, H. R., Hernández, R., Pirker, J., \& Gütl, C. (2016). A practical experience on the use of gamification in MOOC courses as a strategy to increase motivation. In International Workshop on Learning Technology for Education in Cloud., (pp. 139149).

- Nakajima, T. and Lehdonvirta, V. (2013). Designing Motivation Using Persuasive Ambient Mirrors. Personal and Ubiquitous Computing, 17 (1), 107-126.

- Nicholson, S. (2012). A user-centered theoretical framework for meaningful gamification. Paper presented at the Games+ Learning+ Society. 8.0, Madison, USA.

- Overby, K. (2011). "Student-Centered Learning," ESSAI: Vol. 9, Article 32. Available at: https://dc.cod.edu/essai/vol9/iss1/32

- Parry, R., Moseley, A., Gretton, N., Tunstall, R., \& Mobbs, M. (2016). Why MOOCs matter: the consequence of massive open online courses for museums, universities, and their publics. MW2016: Museums and the Web 2016. 


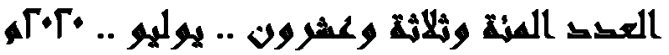

- Pektas, M., \& Kepceoglu, İ. (2019). What Do Prospective Teachers Think about Educational Gamification?. Science Education International, 30(1), 65-74.

- D. H. (2011). Drive. Britain.Canongate Books.

- Rabah, J., Cassidy, R., \& Beauchemin, R. (2018). Gamification in Education: Real Benefits or Edutainment? Proceedings of the European Conference on E-Learning, 489-496. Retrieved from http://search.ebscohost.com.sdl.idm.oclc.org/login.aspx?direct=true\&d $\mathrm{b}=$ ehh\&AN=134951105\&site=eds-live

- Rodriguez, C. O. (2012). MOOCs and the AI-Stanford Like Courses: Two Successful and Distinct Course Formats for Massive Open Online Courses. European Journal of Open, Distance and E-Learning.

- Ross, C. M. (1999). The relationship among academic achievement motivation, motivation orientation, and ability-achievement differences in reading. Unpublished doctoral dissertation, University of Alabama, USA.

- Rouse, K. (2013). Gamification in Science Education: The Relationship of Educational Games to Motivation and Achievement (Unpublished doctoral thesis), The University of Southern Mississippi, United States.

- Rughiniş, R. (2013, June). Gamification for productive interaction: Reading and working with the gamification debate in education. In 2013 8th Iberian Conference on Information Systems and Technologies (CISTI) (pp. 1-5). IEEE.

- Running head: POINTS-BASED REWARD SYSTEMS IN GAMIFICATION, conference paper, 2019. Conference Papers International Communication Association. 2018, p1-26. 26p. Database: Communication \& Mass Media Complete

- Saki, K., \& Nadari, M. (2018). The relationship between self-regulated learning, academic self-concept and the academic achievement motivation of students in the second grade of high school. Middle East Journal of Family Medicine, 16(2), 324. Retrieved from http://search.ebscohost.com.sdl.idm.oclc.org/login.aspx?direct=true\&d $\mathrm{b}=\mathrm{edb} \& \mathrm{AN}=127838482 \&$ site $=$ eds-live

- Saleem, R. Y. (2018). A Suggested Model for Evaluating MOOC Platforms According to Educational and Technical Standards. Life Science Journal, 15(11).

- Sanmugam, M., Abdullah, Z., Mohamed, H., Aris, B., Zaid, N. M., \& Suhadi, S. M. (2016, May). The affiliation between student achievement and elements of gamification in learning science. In 2016 4th International Conference on Information and Communication Technology (ICoICT) (pp. 1-4). IEEE. 


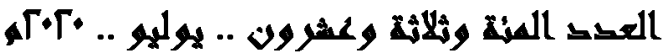

- Saputro, R. E., Salam, S., Zakaria, M. H., \& Anwar, T. (2019). A gamification framework to enhance students' intrinsic motivation on MOOC. TELKOMNIKA, 17(1), 170-178.

- Sepehr, S., \& Head, M. (2013, October). Competition as an element of gamification for learning: an exploratory longitudinal investigation. In Proceedings of the First International Conference on Gameful Design, Research, and Applications (pp. 2-9).

- Skinner, B. F. (1953). Science and Human Behavior. New York, NY: The Free Press.

- Snyman, M.\& Berg, G (2018). The Significance of the Learner Profile in Recognition of Prior Learning. Adult Education Quarterly, Vol. 68(1) $24-40$.

- Subhash, S., \& Cudney, E. A. (2018). Gamified learning in higher education: A systematic review of the literature. Computers in Human Behavior, 87, 192-206.

- Taspinar, B., Schmidt, W., \& Schuhbauer, H. (2016). Gamification in education: a board game approach to knowledge acquisition. Procedia Computer Science, 99, 101-116.

- Terras, M. M., Ramsay, J. (2015). Massive open online courses (MOOCs): Insights and challenges from a psychological perspective. British Journal of Educational Technology, 46(3), 472-487.

- Thoms, B (2011)a dynamic social feedback system to support learning and social interactions in higher education. IEEE Transactions on Learning Technologies, 4(4), 340-352. Retrieved from https:// ieeexplore.ieee.org/document/5740830

- Turan, Z., Avinc, Z., Kara, K., \& Goktas, Y. (2016). Gamification and education: Achievements, cognitive loads, and views of students. International Journal of Emerging Technologies in Learning (iJET), 11(07), 64-69.

- UNESCO. OER development and publishing initiatives. Available online https://en.unesco.org/themes/building-knowledge-societies/ oer

- Van Roy, R., \& Zaman, B. (2018). Need-supporting gamification in education: An assessment of motivational effects over time. Computers \& Education, 127, 283-297.

- Wen, M., Yang, D., \& Rosé, C. P. (2014). Linguistic reflections of student engagement in massive open online courses. In the Eighth International Conference on Weblogs and Social Media. Palo Alto, California.

- Werbach, K., \& Hunter, D. (2015). The gamification toolkit: dynamics, mechanics, and components for the win. Wharton Digital Press Book.

- Wigfield, A., \& Cambria, J. (2010). Achievement motivation. The Corsini Encyclopedia of Psychology, 1-2. 
- Xiong, Y., Li, H., Kornhaber, M. L., Suen, H. K., Pursel, B., \& Goins, D. D. (2015). Examining the relations among student motivation, engagement, and retention in a MOOC: A structural equation modeling approach. Global Education Review, 2(3), 23-33.

- Yang, D., \& Rosé, C. P. (2013).“ Turn on , Tune in , Drop out ": Anticipating student dropouts in Massive Open Online Courses. In NIPS Workshop on Data Driven Education. Lake Tahoe, Nevada.

- Yildirim, I. (2017). The effects of gamification-based teaching practices on student achievement and students' attitudes toward lessons. The Internet and Higher Education, 33, 86-92.

- Yildırım, İ. (2017). Students' Perceptions about Gamification of Education: A Q-Method Analysis. Education \& Science/Egitim ve Bilim, 42(191).

- Yuan, L., \& Powell, S. (2013). MOOCs and open education : Implications for higher education. Bolton: CETIS. Retrieved from http://publications.cetis.ac.uk/2013/667

- Yuan, Li. Sheila, M., \& Wilbert K. (2008). Open Educational Resources Opportunities and Challenges for Higher Education. Briefing paper prepared for the UK Joint Information Systems Committee Centre for Educational Technology \& Interoperability Standards (JISC CETIS).

- Zajonc, R.B. (1965). Social Facilitation. A Solution Suggested for an Old Unresolved social Psychological Problem. Science, 149 (3681), 269-274.

- ZEBING WU. (2019). Academic Motivation, Engagement, and Achievement among College Students. College Student Journal, 53(1), 112. Retrieved from http://search.ebscohost.com.sdl.idm.oclc. org/login.aspx?direct=true\&db=ehh\&AN=136193466\&site=eds-live

- Zichermann, G. and Cunningham, C. (2011). Gamification by Design: Implementing Game Mechanics in Web and Mobile Apps. 1st Edition. O'Reilly Media, Sebastopol, USA

\section{粎料料潾}

